\title{
Signal Constellations for Quasi-Orthogonal Space-Time Block Codes With Full Diversity
}

\author{
Weifeng Su, Member, IEEE, and Xiang-Gen Xia, Senior Member, IEEE
}

\begin{abstract}
Space-time block codes (STBCs) from orthogonal designs proposed by Alamouti, and Tarokh-Jafarkhani-Calderbank have attracted considerable attention lately due to their fast maximum-likelihood (ML) decoding and full diversity. However, the maximum symbol transmission rate of an STBC from complex orthogonal designs for complex signals is only $3 / 4$ for three and four transmit antennas, and it is difficult to construct complex orthogonal designs with rate higher than $1 / 2$ for more than four transmit antennas. Recently, Jafarkhani, Tirkkonen-Boariu-Hottinen, and Papadias-Foschini proposed STBCs from quasi-orthogonal designs, where the orthogonality is relaxed to provide higher symbol transmission rates. With the quasi-orthogonal structure, the quasi-orthogonal STBCs still have a fast ML decoding, but do not have the full diversity. The performance of these codes is better than that of the codes from orthogonal designs at low signal-to-noise ratio (SNR), but worse at high SNR. This is due to the fact that the slope of the performance curve depends on the diversity. It is desired to have the quasi-orthogonal STBCs with full diversity to ensure good performance at high SNR. In this paper, we achieve this goal by properly choosing the signal constellations. Specifically, we propose that half of the symbols in a quasi-orthogonal design are chosen from a signal constellation set $\mathcal{A}$ and the other half of them are chosen from a rotated constellation $e^{j \phi} \mathcal{A}$. The resulting STBCs can guarantee both full diversity and fast ML decoding. Moreover, we obtain the optimum selections of the rotation angles $\phi$ for some commonly used signal constellations. Simulation results show that the proposed codes outperform the codes from orthogonal designs at both low and high SNRs.
\end{abstract}

Index Terms-Diversity, multiple antennas, orthogonal designs, quasi-orthogonal designs, space-time block codes (STBC), wireless communications.

\section{INTRODUCTION}

$\mathbf{T}$ ELATAR [1] and Foschini and Gans [2] have recently shown that there is a huge potential capacity gain of multiple-antenna communication systems compared to single-antenna communication systems. They showed that the capacity of a multiple-antenna system grows at least linearly with the number of transmit antennas, provided that the number

Manuscript received January 25, 2002; revised January 14, 2003. This work was supported in part by the Air Force Office of Scientific Research (AFOSR) under Grant F49620-02-1-0157, and the National Science Foundation under Grants MIP-9703377, CCR-0097240, and CCR-0325180. The material in this paper was presented in part at the IEEE Global Telecommunications Conference, Taipei, Taiwan, R.O.C., December 2002.

W. Su was with the Department of Electrical and Computer Engineering, University of Delaware, Newark, DE 19716 USA. He is now with the Department of Electrical and Computer Engineering, University of Maryland, College Park, MD 20742 USA (e-mail: weifeng@eng.umd.edu).

X.-G. Xia is with the Department of Electrical and Computer Engineering, University of Delaware, Newark, DE 19716 USA (e-mail: xxia@ee.udel.edu).

Communicated by D. N. C. Tse, Associate Editor for Communications.

Digital Object Identifier 10.1109/TIT.2004.834740 of receive antennas is greater than or equal to the number of transmit antennas. To approach the potential huge capacity of the multiple-antenna systems, new codes and modulation signals, which are called space-time codes or space-time signals, have attracted considerable attention lately, see, for example, [3]-[31]. The fundamental performance criteria of space-time codes were derived by Guey, Fitz, Bell, and Kuo [3], and Tarokh, Seshadri, and Calderbank [4]. To achieve the maximum diversity, the difference matrix between any two distinct codewords should be of full rank. Note that, since space-time codes are matrices, even for a small block size of codewords and a reasonable rate, the size of a codebook can be quite large and the maximum-likelihood (ML) decoding may have a considerably high complexity. Therefore, a "good" space-time code should possess two properties: i) full diversity; and ii) fast ML decoding.

One attractive approach of space-time code design is to construct space-time block codes (STBCs) from orthogonal designs as proposed by Alamouti [5] and Tarokh, Jafarkhani, and Calderbank [6]. These codes achieve full diversity and have fast ML decoding at the receiver. The transmitted symbols can be decoded separately, not jointly. Thus, the decoding complexity increases linearly, not exponentially, with the code size. However, the maximum symbol transmission rate of an STBC from orthogonal designs ${ }^{1}$ is only $3 / 4$ for three and four transmit antennas [19] without linear processing and [25] with linear processing (more general) and $(l+1) /(2 l)$ for $2 l-1$ or $2 l$ transmit antennas [21] without linear processing, and it is difficult to construct orthogonal designs with rate higher than $1 / 2$ for more than four transmit antennas [6], [19]-[23].

Recently, Jafarkhani [13], Tirkkonen, Boariu, and Hottinen [16], and Papadias and Foschini [17] proposed STBCs from quasi-orthogonal designs, where the orthogonality is relaxed to provide higher symbol transmission rate. With the quasi-orthogonal structure, the ML decoding at the receiver can be done by searching pairs of symbols, similar to the codes from orthogonal designs where the ML decoding can be done by searching single symbols. However, these codes do not achieve the full diversity. The performance of these codes is better than that of the codes from orthogonal designs at low signal-to-noise ratio (SNR), but worse at high SNR. This is due to the fact that the slope of the performance curve depends on the diversity order.

It is desired to have the STBCs from quasi-orthogonal designs with full diversity to ensure good performance at high SNR. In this paper, based on the quasi-orthogonal code structures in the Jafarkhani scheme, the Papadias-Foschini scheme, and the

${ }^{1}$ For simplicity, orthogonal designs stand for complex orthogonal designs throughout the paper. 
Tirkkonen-Boariu-Hottinen scheme (TBH scheme for short), we introduce a new modulation scheme by properly choosing the signal constellations. Specifically, we propose that half of the symbols in a quasi-orthogonal design are chosen from a signal constellation set $\mathcal{A}$ and the other half of them are chosen from a rotated constellation $e^{j \phi} \mathcal{A}$. The resulting STBCs can guarantee both full diversity and fast ML decoding. In this paper, we also obtain the optimum selections of the rotation angles $\phi$ for some commonly used signal constellations. The decoding complexity and symbol transmission rate of the new scheme are the same as those of the Jafarkhani scheme or the Papadias-Foschini scheme or the TBH scheme. Simulation results show that the new scheme outperforms the codes from orthogonal designs at both low and high SNRs.

This paper is organized as follows. In Section II, we specify the channel model and briefly review the design criteria for space-time codes. In Section III, we briefly review the existing STBCs from orthogonal and quasi-orthogonal designs. In Section IV, based on the Jafarkhani scheme, the Papadias-Foschini scheme, and the TBH scheme, we propose the new modulation scheme by properly choosing the signal constellations. We systematically investigate the signal constellations and determine the optimum rotation angles in Section V. In Section VI, we calculate the diversity product (or coding gain or coding advantage or product distance or minimum determinant) of the quasi-orthogonal STBCs with full diversity for different signal constellations, and provide some simulation results for systems with four and eight transmit antennas. In Section VII, we further discuss the proposed scheme, and propose a more general modulation scheme with full diversity. Finally, we conclude this paper with some comments in Section VIII.

\section{Channel Model AND Design CRITERIA FOR SPACE-TIME CODES}

We consider a wireless communication system with $n$ transmit antennas and $m$ receive antennas. The system can be modeled as

$$
Y=\sqrt{\frac{\rho}{n}} C H+N
$$

where $C=\left\{c_{t}^{i}\right\}_{1 \leq t \leq T, 1 \leq i \leq n}$ is the transmitted signal matrix of size $T \times n$ whose entry $c_{t}^{i}$ is the signal transmitted at antenna $i$ at time $t ; H=\left\{h_{i, j}\right\}$ is the channel coefficient matrix of size $n \times m$ whose entry $h_{i, j}$ is the channel coefficient from transmit antenna $i$ to receive antenna $j ; Y=\left\{y_{t}^{j}\right\}_{1 \leq t \leq T, 1 \leq j \leq m}$. is the received signal matrix of size $T \times m$ whose entry $y_{t}^{j}$ is the signal received at antenna $j$ at time $t$; and $N=\left\{n_{t}^{j}\right\}$ is the noise matrix of size $T \times m$. The noise samples and the entries of $H$ are independent samples of a zero-mean complex Gaussian random variable with variance 1 . The fading channel is quasi-static in the sense that the channel coefficients do not change during one codeword transmission, and change independently from one codeword transmission to the next. Finally, we normalize the transmitted signal matrix $C$ obeying the energy constraint

$$
E\|C\|_{F}^{2}=n T
$$

where $\|C\|_{F}$ is the Frobenius norm ${ }^{2}$ of $C$ and $E$ stands for the expectation. Then, the factor $\sqrt{\rho / n}$ in (1) ensures that $\rho$ is the SNR at each receive antenna, and independent of $n$.

If perfect channel state information is available at the receiver, then the ML decoding is to minimize the decision metric $\min _{C}\left\|Y-\sqrt{\frac{\rho}{n}} C H\right\|_{F}^{2}$ for detecting the transmitted codeword. Note that, in general, the number of $T \times n$ matrices $C$ needed in a codebook is large. If the rate of a system is $R$ bits per channel use, the number of matrices is $2^{R T}$. For the quasi-static and flat Rayleigh-fading channels, the pairwise error probability is upper-bounded as ([3], [4])

$$
P(C \rightarrow \tilde{C}) \leq \frac{1}{2}\left(\prod_{i=1}^{r} \lambda_{i}\right)^{-m} \cdot\left(\frac{\rho}{4 n}\right)^{-r m}
$$

where $r=\operatorname{rank}(C-\tilde{C})$, and $\lambda_{1}, \lambda_{2}, \ldots, \lambda_{r}$ are the nonzero eigenvalues of $(C-\tilde{C})(C-\tilde{C})^{\mathcal{H}}$. Here, the superscript $\mathcal{H}$ stands for the complex conjugate and transpose of a matrix. The upper bound in (2) leads to two design criteria for space-time codes ([3], [4]): i) Rank Criterion or Diversity Criterion: the minimum rank of the difference matrix $C-\tilde{C}$ over all pairs of distinct codewords $C$ and $\tilde{C}$ should be as large as possible; and ii) Product Criterion: the minimum value of the product $\lambda_{1} \lambda_{2} \cdots \lambda_{r}$ over all pairs of distinct codewords $C$ and $\tilde{C}$ should also be as large as possible.

The diversity criterion is the more important one of the two since it determines the slope of the performance curve. In order to achieve the maximum diversity, the difference matrix $C-\tilde{C}$ has to be full rank for any pair of distinct codewords $C$ and $\tilde{C}$. The product criterion is of secondary importance and should be optimized if the full diversity is achieved. If $(C-\tilde{C})(C-$ $\tilde{C})^{\mathcal{H}}$ is of full rank, then the product $\lambda_{1} \lambda_{2} \cdots \lambda_{n}$ is equal to the determinant of $(C-\tilde{C})(C-\tilde{C})^{\mathcal{H}}$. In this case, which implies ( $n \geq T)$, a helpful quantity $\zeta$ called diversity product [10], [12] is given by

$$
\zeta=\frac{1}{2 \sqrt{n}} \min _{C \neq \tilde{C}}\left|\operatorname{det}\left[(C-\tilde{C})(C-\tilde{C})^{\mathcal{H}}\right]\right|^{1 /(2 T)} .
$$

The factor ${ }^{3} \frac{1}{2 \sqrt{n}}$ guarantees that $0 \leq \zeta \leq 1$, since

$$
\begin{aligned}
\min _{C \neq \tilde{C}}\left|\operatorname{det}\left[(C-\tilde{C})(C-\tilde{C})^{\mathcal{H}}\right]\right|^{1 / T} \\
\leq \frac{1}{T} \min _{C \neq \tilde{C}}\|C-\tilde{C}\|_{F}^{2} \\
\leq \frac{1}{T} E\|C-\tilde{C}\|_{F}^{2} \leq 4 n
\end{aligned}
$$

When all codewords are square matrices, i.e., $T=n$, the diversity product can be simplified as

$$
\zeta=\frac{1}{2 \sqrt{n}} \min _{C \neq \tilde{C}}|\operatorname{det}(C-\tilde{C})|^{1 / n} .
$$

${ }^{2}$ The Frobenius norm of $C$ satisfies

$$
\|C\|_{F}^{2}=\operatorname{tr}\left(C^{\mathcal{H}} C\right)=\operatorname{tr}\left(C C^{\mathcal{H}}\right)=\sum_{t=1}^{T} \sum_{i=1}^{n}\left|c_{t}^{i}\right|^{2} .
$$

\footnotetext{
${ }^{3}$ The normalization factor $\frac{1}{2 \sqrt{n}}$ is needed here because the energy constraint is $E\|C\|_{F}^{2}=n T$, while the normalization factor $1 / 2$ was used in [10], [12] because all codewords considered there are $n \times n$ unitary matrices, i.e., the energy constraint is $E\|C\|_{F}^{2}=n$.
} 
Substituting (4) into (2), the pairwise error probability is upperbounded as

$$
P(C \rightarrow \tilde{C}) \leq \frac{1}{2}\left(\zeta^{2} \rho\right)^{-n m}
$$

\section{STBCS FROM ORTHOGONAL OR QUASI-ORTHOGONAL DESIGNS}

In this section, we briefly review the existing STBCs from orthogonal or quasi-orthogonal designs. For more details, we refer the reader to ([5], [6], [13], [16], [17], [19], [21]-[23], [30], [31], the references therein).

\section{A. STBCs From Orthogonal Designs}

An orthogonal design in complex variables $x_{1}, x_{2}, \ldots, x_{k}$ is a $p \times n$ matrix $G\left(x_{1}, x_{2}, \ldots, x_{k}\right)$ such that: i) the entries of $G$ are complex linear combinations of $x_{1}, x_{1}^{*}, x_{2}, x_{2}^{*}, \ldots, x_{k}, x_{k}^{*}$; and ii)

$$
G^{\mathcal{H}} G=\left(\left|x_{1}\right|^{2}+\left|x_{2}\right|^{2}+\cdots+\left|x_{k}\right|^{2}\right) I_{n}
$$

where $I_{n}$ is the $n \times n$ identity matrix. ${ }^{4}$ The rate of $G\left(x_{1}, x_{2}, \ldots, x_{k}\right)$ is defined as $k / p$.

The orthogonal design $G\left(x_{1}, x_{2}, \ldots, x_{k}\right)$ of size $p \times n$ can be used to construct STBCs for $n$ transmit antennas. Specifically, for any $i, 1 \leq i \leq k$, let $\mathcal{A}_{i}$ denote a signal constellation with $2^{b_{i}}$ elements and average energy 1 . Then, for any information sequence of $b_{1}+b_{2}+\cdots+b_{k}$ bits, it is mapped to $k$ constellation symbols $s_{1} \in \mathcal{A}_{1}, s_{2} \in$ $\mathcal{A}_{2}, \ldots, s_{k} \in \mathcal{A}_{k}$. Replacing $x_{1}, x_{2}, \ldots, x_{k}$ everywhere in $G\left(x_{1}, x_{2}, \ldots, x_{k}\right)$ by $s_{1}, s_{2}, \ldots, s_{k}$, respectively, we have the matrix $G\left(s_{1}, s_{2}, \ldots, s_{k}\right)$. Finally, the STBC to be transmitted is

$$
C=\sqrt{\frac{p}{k}} G\left(s_{1}, s_{2}, \ldots, s_{k}\right) .
$$

The factor $\sqrt{p / k}$ ensures that the code obeys the energy constraint, i.e., $E\|C\|_{F}^{2}=n p$. The symbol transmission rate of this code is defined as $k / p$, which means that there are $k$ information symbols transmitted in each codeword with time delay $p$.

For any pair of distinct codewords

$$
\sqrt{p / k} G\left(s_{1}, s_{2}, \ldots, s_{k}\right) \text { and } \sqrt{p / k} G\left(\tilde{s}_{1}, \tilde{s}_{2}, \ldots, \tilde{s}_{k}\right)
$$

the difference matrix is $\sqrt{p / k} G\left(s_{1}-\tilde{s}_{1}, s_{2}-\tilde{s}_{2}, \ldots, s_{k}-\tilde{s}_{k}\right)$, and denoted as $\Delta C$ for simplicity. From (5), we have

$$
(\Delta C)^{\mathcal{H}}(\Delta C)=\frac{p}{k}\left(\left|s_{1}-\tilde{s}_{1}\right|^{2}+\left|s_{2}-\tilde{s}_{2}\right|^{2}+\cdots+\left|s_{k}-\tilde{s}_{k}\right|^{2}\right) I_{n} .
$$

It implies that STBCs from orthogonal designs guarantee full diversity according to the rank criterion. Moreover, by (3), the diversity product is

$$
\zeta=\frac{1}{2 \sqrt{k}} \min \left\{d_{1}, d_{2}, \ldots, d_{k}\right\}
$$

${ }^{4}$ For convenience, in what follows we use the orthogonality (5) while the more general orthogonality $G^{\mathcal{H}} G=\left|x_{1}\right|^{2} D_{1}+\left|x_{2}\right|^{2} D_{2}+\cdots+\left|x_{k}\right|^{2} D_{k}$ also applies [6] where $D_{1}, \ldots, D_{k}$ are $k$ constant diagonal matrices with positive diagonal elements. where $d_{1}, d_{2}, \ldots, d_{k}$ are the minimum Euclidean distances of the signal constellations $\mathcal{A}_{1}, \mathcal{A}_{2}, \ldots, \mathcal{A}_{k}$, respectively. If all of $\mathcal{A}_{i}$ are $r$-PSK, then the diversity product is $\zeta=1 / \sqrt{k} \sin (\pi / r)$.

The first STBC from orthogonal design was proposed by Alamouti [5] for two transmit antennas. It is due to a $2 \times 2$ orthogonal design of rate 1

$$
G_{2}\left(x_{1}, x_{2}\right)=\left[\begin{array}{rr}
x_{1} & x_{2} \\
-x_{2}^{*} & x_{1}^{*}
\end{array}\right]
$$

For three and four transmit antennas, there are codes of rate $3 / 4$ [9], [14], [15]:

$$
\begin{aligned}
G_{3}\left(x_{1}, x_{2}, x_{3}\right) & =\left[\begin{array}{rrr}
x_{1} & x_{2} & x_{3} \\
-x_{2}^{*} & x_{1}^{*} & 0 \\
-x_{3}^{*} & 0 & x_{1}^{*} \\
0 & -x_{3}^{*} & x_{2}^{*}
\end{array}\right] \\
G_{4}\left(x_{1}, x_{2}, x_{3}\right) & =\left[\begin{array}{rrrr}
x_{1} & x_{2} & x_{3} & 0 \\
-x_{2}^{*} & x_{1}^{*} & 0 & x_{3} \\
-x_{3}^{*} & 0 & x_{1}^{*} & -x_{2} \\
0 & -x_{3}^{*} & x_{2}^{*} & x_{1}
\end{array}\right] .
\end{aligned}
$$

For $n=2^{l}$ transmit antennas, there are several realizations of orthogonal designs of square size with rate $(l+1) / 2^{l}$ from different approaches [32], [33], [14], [15], [35]. All of these realizations are equivalent. An intuitive interpretation of these realizations was given in [19] as follows. Let $G_{1}\left(x_{1}\right)=x_{1} I_{1}$, and

$$
\begin{aligned}
& G_{2^{l}}\left(x_{1}, x_{2}, \ldots, x_{l+1}\right) \\
& \quad=\left[\begin{array}{cc}
G_{2^{l-1}}\left(x_{1}, x_{2}, \ldots, x_{l}\right) & x_{l+1} I_{2^{l-1}} \\
-x_{l+1}^{*} I_{2^{l-1}} & G_{2^{l-1}}^{\mathcal{H}}\left(x_{1}, x_{2}, \ldots, x_{l}\right)
\end{array}\right]
\end{aligned}
$$

for $l=1,2,3, \ldots$. For some number of transmit antennas which is not a power of 2 , orthogonal designs can be obtained from (11) by truncating some columns. For orthogonal designs of nonsquare size, Tarokh, Jafarkhani, and Calderbank in [6] provided a design method with rate $1 / 2$ for any number of transmit antennas. Recently, systematic design methods were independently proposed in [21]-[23] to generate orthogonal designs of rates $(l+1) /(2 l)$ for $2 l-1$ or $2 l$ transmit antennas. The designs in [21], [22] do not have closed forms and the designs in [23] have closed forms and, furthermore, in [23] a design with half delays of the ones in [21], [22] is proposed when the number of transmit antennas is a multiple of 4 , such as $4,8,12$, etc.

\section{B. STBCs From Quasi-Orthogonal Designs}

To improve the symbol transmission rate, one natural way is to relax the requirement of the orthogonality in STBCs. In [13], Jafarkhani proposed STBCs from quasi-orthogonal designs. A similar scheme was also proposed by Papadias-Foschini in [17].

For four transmit antennas, a quasi-orthogonal STBC with symbol transmission rate 1 was constructed from the Alamouti scheme as follows [13]:

$$
C=\left[\begin{array}{rr}
A & B \\
-\bar{B} & \bar{A}
\end{array}\right]=\left[\begin{array}{rrrr}
x_{1} & x_{2} & x_{3} & x_{4} \\
-x_{2}^{*} & x_{1}^{*} & -x_{4}^{*} & x_{3}^{*} \\
-x_{3}^{*} & -x_{4}^{*} & x_{1}^{*} & x_{2}^{*} \\
x_{4} & -x_{3} & -x_{2} & x_{1}
\end{array}\right]
$$


where

$$
A=\left[\begin{array}{rr}
x_{1} & x_{2} \\
-x_{2}^{*} & x_{1}^{*}
\end{array}\right], \quad B=\left[\begin{array}{rr}
x_{3} & x_{4} \\
-x_{4}^{*} & x_{3}^{*}
\end{array}\right]
$$

and $\bar{A}$ and $\bar{B}$ are the complex conjugate, not transpose, of $A$ and $B$, respectively. One can check that

$$
C^{\mathcal{H}} C=\left[\begin{array}{rrrr}
a & 0 & 0 & b \\
0 & a & -b & 0 \\
0 & -b & a & 0 \\
b & 0 & 0 & a
\end{array}\right]
$$

where

$$
a=\left|x_{1}\right|^{2}+\left|x_{2}\right|^{2}+\left|x_{3}\right|^{2}+\left|x_{4}\right|^{2}
$$

and

$$
b=x_{1} x_{4}^{*}+x_{4} x_{1}^{*}-x_{2} x_{3}^{*}-x_{3} x_{2}^{*} .
$$

We can see that the ML decision metric of this code can be written as the sum of two terms $f_{1}\left(s_{1}, s_{4}\right)+f_{2}\left(s_{2}, s_{3}\right)$, where $f_{1}$ depends only on $s_{1}$ and $s_{4}$, and $f_{2}$ depends only on $s_{2}$ and $s_{3}$. Thus, the minimization can be done separately on these two terms, i.e., symbol pairs $\left(s_{1}, s_{4}\right)$ and $\left(s_{2}, s_{3}\right)$ can be decoded separately, which leads to fast ML decoding. However, according to (14), the minimum rank of the difference matrix between two distinct codewords is 2 , which means that the code (12) does not have full diversity. Note that there are other code structures ${ }^{5}$ which can provide behaviors similar to those of (12) and they do not have the full diversity like (12). For eight transmit antennas, a code with rate $3 / 4$ was also constructed, compared with the orthogonal STBCs where the rate is only $1 / 2$.

In [16], Tirkkonen, Boariu, and Hottinen proposed a similar scheme (TBH scheme for short) for quasi-orthogonal STBCs. For four transmit antennas, the TBH scheme is

$$
C=\left[\begin{array}{ll}
A & B \\
B & A
\end{array}\right]=\left[\begin{array}{rrrr}
x_{1} & x_{2} & x_{3} & x_{4} \\
-x_{2}^{*} & x_{1}^{*} & -x_{4}^{*} & x_{3}^{*} \\
x_{3} & x_{4} & x_{1} & x_{2} \\
-x_{4}^{*} & x_{3}^{*} & -x_{2}^{*} & x_{1}^{*}
\end{array}\right]
$$

where $A$ and $B$ are the same as those in (13). Similarly

$$
C^{\mathcal{H}} C=\left[\begin{array}{cccc}
a & 0 & b & 0 \\
0 & a & 0 & b \\
b & 0 & a & 0 \\
0 & b & 0 & a
\end{array}\right]
$$

where

$$
a=\left|x_{1}\right|^{2}+\left|x_{2}\right|^{2}+\left|x_{3}\right|^{2}+\left|x_{4}\right|^{2}
$$

and

$$
b=x_{1} x_{3}^{*}+x_{3} x_{1}^{*}-x_{2} x_{4}^{*}-x_{4} x_{2}^{*} .
$$

The behaviors of (15) are similar to those of (12). For eight transmit antennas, a code with rate 1 was also constructed [16]. However, for eight transmitted symbols $s_{1}, s_{2}, \ldots, s_{8}$, this

${ }^{5}$ Note that, with the structure

$$
\left[\begin{array}{rr}
A & B \\
-B & A
\end{array}\right]
$$

mentioned in [13], the transmitted symbols $s_{1}, s_{2}, s_{3}$ and $s_{4}$ cannot be separated into two groups at the receiver for the fast ML decoding. code can separate them only into two groups: $\left(s_{1}, s_{3}, s_{5}, s_{7}\right)$ and $\left(s_{2}, s_{4}, s_{6}, s_{8}\right)$. Therefore, one cannot decode them pairs by pairs.

\section{QUASI-ORTHOGONAL STBCs With FULl DiVERSITY}

The performance of the quasi-orthogonal STBCs code is better than that of the codes from orthogonal designs at low SNR, but worse at high SNR. As mentioned in [13], [16], this is due to the fact that the slope of the performance curve depends on the diversity. In fact, in the Jafarkhani scheme [13], the Papadias-Foschini scheme [17], and the TBH scheme [16], the signal constellations are chosen arbitrarily. With such a way to select the information symbols, the resulting STBCs cannot guarantee full diversity. The main idea of our new scheme is to choose the signal constellations properly to ensure that the resulting codes achieve full diversity. In the following, we focus on the TBH scheme. The discussion for the Jafarkhani scheme and the Papadias-Foschini scheme is similar.

Assume that $G_{p}\left(x_{1}, x_{2}, \ldots, x_{k}\right)$ is a $p \times p$ orthogonal design in complex variables $x_{1}, x_{2}, \ldots, x_{k}$. A quasi-orthogonal design $Q_{2 p}\left(x_{1}, x_{2}, \ldots, x_{2 k}\right)$ of size $2 p \times 2 p$ in complex variables $x_{1}, x_{2}, \ldots, x_{2 k}$ is defined as

$$
Q_{2 p}=\left[\begin{array}{cc}
A & B \\
B & A
\end{array}\right]
$$

where

$$
A=G_{p}\left(x_{1}, x_{2}, \ldots, x_{k}\right)
$$

and

$$
B=G_{p}\left(x_{k+1}, x_{k+2}, \ldots, x_{2 k}\right) .
$$

Since both $A$ and $B$ are orthogonal designs, we have

$$
\begin{aligned}
Q_{2 p}^{\mathcal{H}} Q_{2 p} & =\left[\begin{array}{ll}
A^{\mathcal{H}} A+B^{\mathcal{H}} B & A^{\mathcal{H}} B+B^{\mathcal{H}} A \\
B^{\mathcal{H}} A+A^{\mathcal{H}} B & B^{\mathcal{H}} B+A^{\mathcal{H}} A
\end{array}\right] \\
& =\left[\begin{array}{ll}
a I_{p} & b I_{p} \\
b I_{p} & a I_{p}
\end{array}\right]
\end{aligned}
$$

where

$$
a=\sum_{i=1}^{2 k}\left|x_{i}\right|^{2} \text { and } b=\sum_{i=1}^{k}\left(x_{i} x_{k+i}^{*}+x_{k+i} x_{i}^{*}\right) .
$$

The last equality in (18) follows from

$$
A^{\mathcal{H}} A+B^{\mathcal{H}} B=\left(\sum_{i=1}^{k}\left|x_{i}\right|^{2}\right) I_{p}+\left(\sum_{i=1}^{k}\left|x_{k+i}\right|^{2}\right) I_{p}
$$

and

$$
\begin{aligned}
A^{\mathcal{H}} B+B^{\mathcal{H}} A= & (A+B)^{\mathcal{H}}(A+B)-A^{\mathcal{H}} A-B^{\mathcal{H}} B \\
= & \left(\sum_{i=1}^{k}\left|x_{i}+x_{k+i}\right|^{2}\right) I_{p}-\left(\sum_{i=1}^{k}\left|x_{i}\right|^{2}\right) I_{p} \\
& -\left(\sum_{i=1}^{k}\left|x_{k+i}\right|^{2}\right) I_{p} .
\end{aligned}
$$

Now we apply the quasi-orthogonal design $Q_{2 p}\left(x_{1}, x_{2}, \ldots, x_{2 k}\right)$ to construct STBCs with full diversity for $2 p$ transmit antennas. For each $i, 1 \leq i \leq k$, let $\mathcal{A}_{i}$ denote a signal constellation with average energy 1 , and $\mathcal{A}_{k+i}$ denote the signal constellation generated by rotating $\mathcal{A}_{i}$ with an angle of $\phi_{i}$, i.e., $\mathcal{A}_{k+i}=\left\{e^{j \phi_{i}} s: s \in \mathcal{A}_{i}\right\}$, denoted as 
$e^{j \phi_{i}} \mathcal{A}_{i}$. Furthermore, let $\left|\mathcal{A}_{i}\right|$ denote the number of elements in $\mathcal{A}_{i}$. For any information sequence of $\log _{2}\left(\prod_{i=1}^{2 k}\left|\mathcal{A}_{i}\right|\right)$ bits, it chooses $2 k$ symbols $s_{1} \in \mathcal{A}_{1}, s_{2} \in \mathcal{A}_{2}, \ldots, s_{2 k} \in \mathcal{A}_{2 k}$. Replacing $x_{1}, x_{2}, \ldots, x_{2 k}$ everywhere in $Q_{2 p}\left(x_{1}, x_{2}, \ldots, x_{2 k}\right)$ by $s_{1}, s_{2}, \ldots, s_{2 k}$, respectively, we have the matrix $Q_{2 p}\left(s_{1}, s_{2}, \ldots, s_{2 k}\right)$. Finally, the STBC to be transmitted is

$$
C=\sqrt{\gamma} Q_{2 p}\left(s_{1}, s_{2}, \ldots, s_{2 k}\right) \text {. }
$$

The factor $\sqrt{\gamma}$ ensures that the transmitted signal in (19) obeys the energy constraint. We will specify $\sqrt{\gamma}$ later. The rate of this code is $\frac{1}{2 p} \log _{2}\left(\prod_{i=1}^{2 k}\left|\mathcal{A}_{i}\right|\right)$ bits per channel use. According to (18), the ML decoding of (19) can be done separately on each pair of symbols $s_{i}$ and $s_{k+i}$.

For any pair of distinct transmitted signals $C=$ $\sqrt{\gamma} Q_{2 p}\left(s_{1}, s_{2}, \ldots, s_{2 k}\right)$ and $\tilde{C}=\sqrt{\gamma} Q_{2 p}\left(\tilde{s}_{1}, \tilde{s}_{2}, \ldots, \tilde{s}_{2 k}\right)$, the difference matrix is $\sqrt{\gamma} Q_{2 p}\left(s_{1}-\tilde{s}_{1}, s_{2}-\tilde{s}_{2}, \ldots, s_{2 k}-\tilde{s}_{2 k}\right)$, denoted as $\Delta C$ for simplicity. Then, from (18), we have

$$
(\Delta C)^{\mathcal{H}}(\Delta C)=\gamma\left[\begin{array}{ll}
(\Delta a) I_{p} & (\Delta b) I_{p} \\
(\Delta b) I_{p} & (\Delta a) I_{p}
\end{array}\right]
$$

where

$$
\Delta a=\sum_{i=1}^{2 k}\left|s_{i}-\tilde{s}_{i}\right|^{2}
$$

and

$$
\Delta b=\sum_{i=1}^{k}\left[\left(s_{i}-\tilde{s}_{i}\right)\left(s_{k+i}-\tilde{s}_{k+i}\right)^{*}+\left(s_{k+i}-\tilde{s}_{k+i}\right)\left(s_{i}-\tilde{s}_{i}\right)^{*}\right] .
$$

The determinant of (20) can be calculated as

$$
\operatorname{det}\left[(\Delta C)^{\mathcal{H}}(\Delta C)\right]=\gamma^{2 p}\left[(\Delta a)^{2}-(\Delta b)^{2}\right]^{p} .
$$

Note that, with the Jafarkhani scheme or the Papadias-Foschini scheme or the TBH scheme, i.e., $\phi_{i}=0$ for all $i$, the determinant in (21) can be zero, for example, when $s_{i}-\tilde{s}_{i}=s_{k+i}-\tilde{s}_{k+i}$, which means that the space-time signals do not have the full diversity. Now we can properly choose the rotation angle $\phi_{i}$ to ensure that the determinant in (21) is nonzero.

For example, let us consider a simple case. If all of $\mathcal{A}_{i}, 1 \leq$ $i \leq k$, are binary phase-shift keying (BPSK), i.e., $\mathcal{A}_{i}=\{1,-1\}$, then we choose the rotation angle as $\pi / 2$, i.e., $\phi_{i}=\pi / 2$. The resulting signal constellation $\mathcal{A}_{k+i}=e^{j \pi / 2} \mathcal{A}_{i}=\{j,-j\}$. Therefore, for any two symbols $s_{i}$ and $\tilde{s}_{i}$ in $\mathcal{A}_{i}$, the difference $s_{i}-$ $\tilde{s}_{i}$ belongs to set $\{0,2,-2\}$; and for any two symbols $s_{k+i}$ and $\tilde{s}_{k+i}$ in $\mathcal{A}_{k+i}$, the difference $s_{k+i}-\tilde{s}_{k+i}$ belongs to set $\{0,2 j,-2 j\}$. It is easy to check that $\Delta b$ in (20) is zero. Thus, the determinant of $(\Delta C)^{\mathcal{H}}(\Delta C)$ is nonzero, which means that the space-time signals achieve full diversity.

For other signal constellations, we systematically and optimally investigate the rotation angle in the next section. Furthermore, it is desired to have the diversity product as large as possible if full diversity has been achieved. According to (3) and (21), the diversity product can be calculated as

$$
\begin{aligned}
\zeta & =\frac{1}{2 \sqrt{2 p}} \min _{\Delta C \neq 0}\left|\operatorname{det}\left[(\Delta C)^{\mathcal{H}}(\Delta C)\right]\right|^{1 /(4 p)} \\
& =\frac{1}{2} \sqrt{\frac{\gamma}{2 p}} \min _{1 \leq i \leq k} \min _{\substack{u, \tilde{u} \in \mathcal{A}_{i} ; v, \tilde{v} \in \mathcal{A}_{k+i} \\
(u, v) \neq(\tilde{u}, \tilde{v})}}\left|(u-\tilde{u})^{2}-(v-\tilde{v})^{2}\right|^{1 / 2} .
\end{aligned}
$$

For convenience, let us define the minimum $\zeta$-distance between two signal constellations $\mathcal{A}$ and $\mathcal{B}$ as follows:

$$
d_{\min , \zeta}(\mathcal{A}, \mathcal{B}) \triangleq \min _{\left(s_{1}, s_{2}\right) \neq\left(\tilde{s}_{1}, \tilde{s}_{2}\right)}\left|\left(s_{1}-\tilde{s}_{1}\right)^{2}-\left(s_{2}-\tilde{s}_{2}\right)^{2}\right|^{1 / 2}
$$

where $s_{1}, \tilde{s}_{1}$ and $s_{2}, \tilde{s}_{2}$ are understood as $s_{1}, \tilde{s}_{1} \in \mathcal{A}$, and $s_{2}, \tilde{s}_{2} \in \mathcal{B}$. Obviously, we have

$$
d_{\min , \zeta}(\mathcal{A}, \mathcal{B}) \leq \min \left\{d_{\min }(\mathcal{A}), d_{\min }(\mathcal{B})\right\}
$$

where $d_{\min }(\mathcal{A})$ and $d_{\min }(\mathcal{B})$ are the minimum Euclidean distances of the signal constellations $\mathcal{A}$ and $\mathcal{B}$, respectively. Thus, we have

$$
d_{\min , \zeta}\left(\mathcal{A}, e^{j \phi} \mathcal{A}\right) \leq d_{\min }(\mathcal{A}) .
$$

We now go back to (22). The diversity product can be rewritten as

$$
\begin{aligned}
\zeta & =\frac{1}{2} \sqrt{\frac{\gamma}{2 p}} \min _{1 \leq i \leq k} d_{\min , \zeta}\left(\mathcal{A}_{i}, \mathcal{A}_{k+i}\right) \\
& \leq \frac{1}{2} \sqrt{\frac{\gamma}{2 p}} \min _{1 \leq i \leq k} d_{\min }\left(\mathcal{A}_{i}\right) .
\end{aligned}
$$

We observe that the diversity product is determined by the minimum $\zeta$-distance of each pair of signal constellations $\mathcal{A}_{i}$ and $\mathcal{A}_{k+i}$, and the minimum $\zeta$-distance is upper-bounded by the minimum Euclidean distance of each signal constellation.

In the example we discussed before, where all of signal constellations $\mathcal{A}_{i}$ are BPSK and the rotation angle is chosen as $\pi / 2$, the minimum $\zeta$-distance between $\mathcal{A}_{i}$ and $e^{j \pi / 2} \mathcal{A}_{i}$ is equal to the minimum Euclidean distance of $\mathcal{A}_{i}$. However, for a general signal constellation, $d_{\min }(\mathcal{A})$ is not always achieved by $d_{\min , \zeta}\left(\mathcal{A}, e^{j \phi} \mathcal{A}\right)$. We will show later that if $\mathcal{A}$ is 8 -PSK, then the minimum $\zeta$-distance $d_{\min , \zeta}\left(\mathcal{A}, e^{j \phi} \mathcal{A}\right)$ cannot be greater than $\left(2 \sin \frac{\pi}{8}\right)^{1 / 2} d_{\min }(\mathcal{A})$, which is strictly less than $d_{\min }(\mathcal{A})$.

There are two problems remaining. First, for a fixed-signal constellation $\mathcal{A}$, how to choose the rotation angle $\phi$ such that the minimum $\zeta$-distance between $\mathcal{A}$ and $e^{j \phi} \mathcal{A}$ is as large as possible? Second, how to design the signal constellation $\mathcal{A}$ such that the minimum $\zeta$-distance between $\mathcal{A}$ and $e^{j \phi} \mathcal{A}$ is as large as possible? We answer these two questions completely in the next section. As for the first question, for any fixed-signal constellation $\mathcal{A}$, we can always find the optimum rotation angle $\phi$ by computer search. However, we will give some analytic results on the optimum rotation angle in the next section.

\section{Minimum $\zeta$-Distance and Optimum Rotation Angle}

In this section, we systematically investigate the minimum $\zeta$-distance between $\mathcal{A}$ and $e^{j \phi} \mathcal{A}$ for some commonly used signal constellations $\mathcal{A}$, and determine the optimum rotation angle $\phi$ accordingly. For convenience, we will simplify the notation of the minimum $\zeta$-distance $d_{\min , \zeta}\left(\mathcal{A}, e^{j \phi} \mathcal{A}\right)$ as $d_{\min , \zeta}(\mathcal{A}, \phi)$ or $d_{\min , \zeta}(\phi)$, and simplify the notation of the minimum Euclidean distance $d_{\min }(\mathcal{A})$ as $d_{\min }$.

From (25), we know that the minimum $\zeta$-distance between $\mathcal{A}$ and $e^{j \phi} \mathcal{A}$ is upper-bounded by the minimum Euclidean distance of $\mathcal{A}$, i.e.,

$$
d_{\min , \zeta}(\phi) \leq d_{\min }
$$


For some signal constellation $\mathcal{A}$, we can properly choose the rotation angle $\phi$ such that the equality holds in (27); and for some signal constellations, the equality in (27) can never be achieved.

At first, let us derive a necessary condition for the equality in (27) to hold. For a fixed-signal constellation $\mathcal{A}$, assume $s_{b}$ and $s_{e}$ are two signals in $\mathcal{A}$ such that the Euclidean distance between $s_{b}$ and $s_{e}$ is the minimum Euclidean distance of $\mathcal{A}$, i.e., $\left|s_{b}-s_{e}\right|=d_{\text {min }}$. From (23), we have

$$
\begin{aligned}
d_{\min , \zeta}\left(\mathcal{A}, e^{j \phi} \mathcal{A}\right) & \leq\left|\left(s_{b}-s_{e}\right)^{2}-\left(e^{j \phi} s_{b}-e^{j \phi} s_{e}\right)^{2}\right|^{1 / 2} \\
& =\left|1-e^{j 2 \phi}\right|^{1 / 2} \cdot\left|s_{b}-s_{e}\right| \\
& =|2 \sin \phi|^{1 / 2} d_{\min } .
\end{aligned}
$$

Thus, we can conclude that the minimum $\zeta$-distance between $\mathcal{A}$ and $e^{j \phi} \mathcal{A}$ cannot be greater than $|2 \sin \phi|^{1 / 2} d_{\text {min }}$, i.e.,

$$
d_{\min , \zeta}\left(\mathcal{A}, e^{j \phi} \mathcal{A}\right) \leq|2 \sin \phi|^{1 / 2} d_{\min }
$$

A necessary condition for the equality in (27) to hold is $|2 \sin \phi|^{1 / 2} \geq 1$, i.e., $|\sin \phi| \geq 1 / 2$ or $\pi / 6 \leq|\phi| \leq 5 \pi / 6$. If the signal constellation $\mathcal{A}$ is $r$-PSK, then the effective rotation angle is in the interval $[-\pi / r, \pi / r]$ from the symmetry of signals in $r$-PSK. According to (28), we have

$$
d_{\min , \zeta}\left(\mathcal{A}, e^{j \phi} \mathcal{A}\right) \leq|2 \sin (\pi / r)|^{1 / 2} d_{\min } .
$$

Thus, for $r$-PSK with $r>6$, the minimum $\zeta$-distance is strictly less than $d_{\text {min }}$.

Since the minimum $\zeta$-distance $d_{\min , \zeta}\left(\mathcal{A}, e^{j \phi} \mathcal{A}\right)$ is upper-bounded by the minimum Euclidean distance of $\mathcal{A}$, it is desired to have the signal constellation $\mathcal{A}$ with $d_{\min }$ as large as possible and meanwhile the minimum $\zeta$-distance between $\mathcal{A}$ and $e^{j \phi} \mathcal{A}$ achieving $d_{\text {min }}$. In the following, we propose two classes of signal constellations which possess the desired properties.

We first propose a class of quadrature amplitude modulation (QAM) ${ }^{6}$ constellations.

Theorem 1: Assume that $\mathcal{A}$ be a signal constellation drawn from a square lattice, where the side length of the squares in the lattice is equal to $d_{\min }(\mathcal{A})$. Then, the minimum $\zeta$-distance between $\mathcal{A}$ and $e^{j \pi / 4} \mathcal{A}$ is $d_{\min }(\mathcal{A})$, i.e.,

$$
d_{\min , \zeta}\left(\mathcal{A}, e^{j \pi / 4} \mathcal{A}\right)=d_{\min }(\mathcal{A}) .
$$

In Theorem 1 , the signal constellation $\mathcal{A}$ can be any arbitrary subset of the square lattice. The only requirement is that the side length of the squares in the lattice is equal to $d_{\min }(\mathcal{A})$. A proof of Theorem 1 appears in Appendix A. Therefore, for any signal constellation $\mathcal{A}$ drawn from a square lattice, $\phi=\pi / 4$ is an optimum rotation angle. Note that, the optimum rotation angle here is not unique. For example, for the constellation BPSK we discussed in the previous section, $\phi=\pi / 2$ is an optimum rotation angle. On the other hand, BPSK can be viewed as a constellation drawn from a square lattice, so $\phi=\pi / 4$ is also an optimum rotation angle. Actually, BPSK can also be viewed

\footnotetext{
${ }^{6}$ Throughout the paper, QAM stands for a constellation drawn from a square lattice, where the side length of the squares in the lattice is equal to the minimum Euclidean distance of the constellation.
}

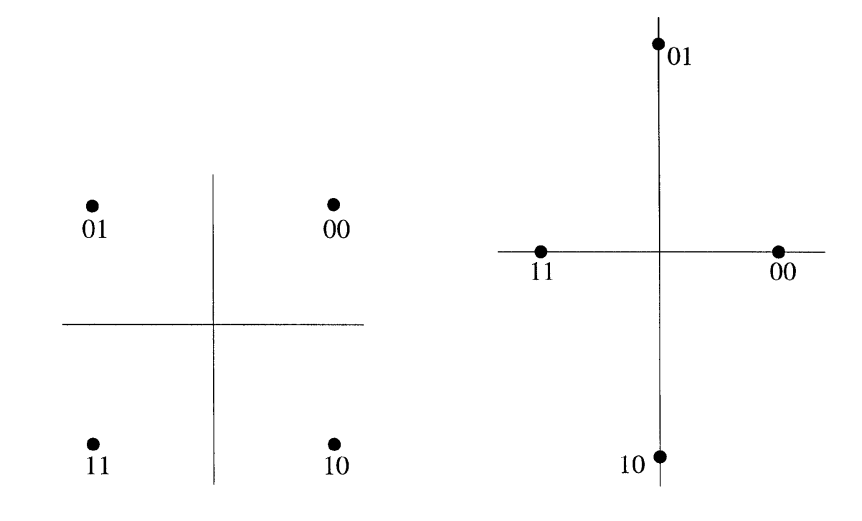

$\begin{array}{ll}\text { (a) } 4-\mathrm{QAM}: d_{\min , \zeta}(\pi / 4)=d_{\min }=\sqrt{2} & \text { (b) } 4-\mathrm{TRI}: d_{\min , \zeta}(\pi / 6)=d_{\min }=\sqrt{2}\end{array}$

Fig. 1. Four-point signal constellations: (a) 4-QAM; (b) 4-TRI.

as a constellation drawn from a lattice of equilateral triangles, thus, $\phi=\pi / 6$ is another optimum rotation angle as stated in the following theorem.

Theorem 2: Assume that $\mathcal{A}$ is a signal constellation drawn from a lattice of equilateral triangles, where the side length of the equilateral triangles is equal to $d_{\min }(\mathcal{A})$. Then, the minimum $\zeta$-distance between $\mathcal{A}$ and $e^{j \pi / 6} \mathcal{A}$ is $d_{\min }(\mathcal{A})$, i.e.,

$$
d_{\min , \zeta}\left(\mathcal{A}, e^{j \pi / 6} \mathcal{A}\right)=d_{\min }(\mathcal{A}) .
$$

A proof of Theorem 2 can be found in Appendix B. The signal constellation $\mathcal{A}$ could be any subset of the lattice of equilateral triangles with the requirement that the side length of the equilateral triangles is equal to $d_{\min }(\mathcal{A})$. For such a signal constellation $\mathcal{A}, \phi=\pi / 6$ is an optimum rotation angle.

Note that for any number of points in two dimensions, the best constellations known by now, from a minimum Euclidean distance point of view, are drawn from the lattices of equilateral triangles [37]-[40]. Moreover, Wayner in [38] proved that for a large number of points, the optimum constellation tends toward a lattice of equilateral triangles.

In the remainder of this section, we calculate the minimum $\zeta$-distance explicitly for different 4-, 8-, and 16-point signal constellations. The calculation for signal constellations of other sizes is similar. We also consider the subject of bit labeling. For convenience, for a signal constellation of $r$ points drawn from a square lattice, we denote it as $r$-QAM; and for a signal constellation of $r$ points drawn from a lattice of equilateral triangles, we denote it as $r$-TRI.

Four-Point Constellations: If the signal constellation $\mathcal{A}$ is chosen as 4-QAM in Fig. 1(a), then according to Theorem 1, $\pi / 4$ is an optimum rotation angle, and the minimum $\zeta$-distance achieves the minimum Euclidean distance. Moreover, with Gray labeling, the difference between the nearest two signals is only 1 bit. The minimum Euclidean distance of 4-TRI in Fig. 1(b) is the same as that of 4-QAM in Fig. 1(a). According to Theorem $2, \pi / 6$ is an optimum rotation angle for 4 -TRI. Because of the triangular nature of 4-TRI, it is impossible to do bit labeling such that the difference between the nearest two signals is always 1 bit [37]. It is not difficult to see that the average bit errors between the nearest two signals in 4-TRI are 1.2 bits. Thus, 4-QAM is the right choice for four-point constellations. 

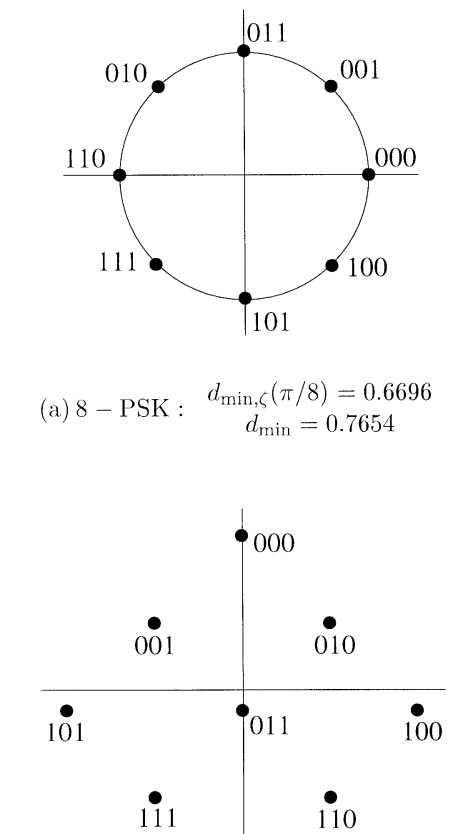

(c) $8-\mathrm{QAM}: d_{\min , \zeta}(\pi / 4)=d_{\min }=0.9562$

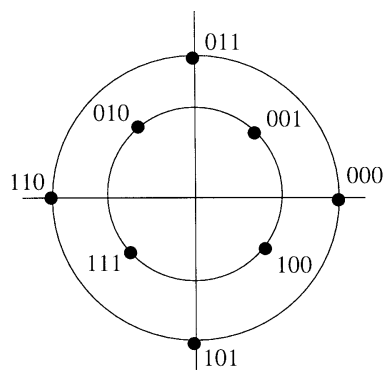

(b) 8 - APSK : $\begin{gathered}d_{\min , \zeta}(\pi / 4)=0.6796 \\ d_{\min }=0.9194\end{gathered}$

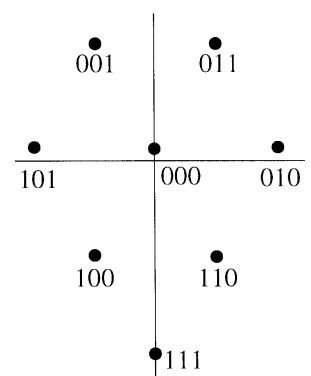

(d) $8-\mathrm{TRI}: d_{\min , \zeta}(\pi / 6)=d_{\min }=0.9631$

Fig. 2. Eight-point signal constellations: (a) 8-PSK; (b) APSK; (c) 8-QAM; (d) 8-TRI.

Eight-Point Constellations: If the signal constellation $\mathcal{A}$ is chosen as 8-PSK in Fig. 2(a), then from (28) we know that the minimum $\zeta$-distance is strictly less than $d_{\min }$. By computer search, the best rotation angle is $\pi / 8$ and the minimum $\zeta$-distance is only 0.6696 . The advantage of 8 -PSK is that with Gray labeling, the difference between the nearest two signals is 1 bit. For signal constellation 8-APSK in Fig. 2(b), by computer search, the best rotation angle is $\pi / 4$, and the minimum $\zeta$-distance is only 0.6796 which is far away from the minimum Euclidean distance 0.9194. Moreover, with bit labeling, the average bit errors between the nearest two signals are 1.33 bits. For signal constellation 8-QAM in Fig. 2(c), according to Theorem $1, \pi / 4$ is an optimum rotation angle, and the minimum $\zeta$-distance achieves the minimum Euclidean distance. With bit labeling, the average bit errors between the nearest two signals are 1.2 bits.

For eight-point constellations, 8-TRI in Fig. 2(d) is the best known constellation from a minimum Euclidean distance point of view. If the signal constellation $\mathcal{A}$ is chosen as 8-TRI, then according to Theorem $2, \pi / 6$ is an optimum rotation angle. However, with bit labeling, the average bit errors between the nearest two signals are 1.29 bits. The overall bit-error rate (BER) performance of 8-TRI is worse than that of 8-QAM.

16-Point Constellations: If the signal constellation $\mathcal{A}$ is chosen as 16-QAM in Fig. 3(a), then according to Theorem 1, $\pi / 4$ is an optimum rotation angle, and the minimum $\zeta$-distance achieves the minimum Euclidean distance. Moreover, with Gray labeling, the difference between the nearest two signals is only 1 bit. For 16-point constellations, 16-TRI in Fig. 3(b) is the best known constellation from a minimum Euclidean distance point of view, which is well known as Voronoi code [38]-[40].
According to Theorem 2, $\pi / 6$ is an optimum rotation angle for 16-TRI. However, the average bit errors between the nearest two signals in 16-TRI are 1.33 bits [37]. Simulation results in the next section show that the performance of 16-TRI is worse than that of 16-QAM. Thus, 16-QAM is the right choice for 16-point constellations.

\section{Diversity Product and Simulation Results}

In this section, we calculate the diversity product (coding gain) for the quasi-orthogonal STBCs with full diversity for different signal constellations, and provide some simulation results for systems with four and eight transmit antennas, respectively. In all simulations, the perfect channel state information is known at the receiver. The channel coefficients are constant during one block of code transmission, and independent from block to block. We compare performances of different schemes in terms of BER.

\section{A. For Four Transmit Antennas}

For four transmit antennas, the quasi-orthogonal STBC with full diversity is given by

$$
C=\left[\begin{array}{rrrr}
x_{1} & x_{2} & x_{3} & x_{4} \\
-x_{2}^{*} & x_{1}^{*} & -x_{4}^{*} & x_{3}^{*} \\
x_{3} & x_{4} & x_{1} & x_{2} \\
-x_{4}^{*} & x_{3}^{*} & -x_{2}^{*} & x_{1}^{*}
\end{array}\right]
$$

where $x_{1}, x_{2} \in \mathcal{A}$ and $x_{3}, x_{4} \in e^{j \phi} \mathcal{A}$ for some signal constellation $\mathcal{A}$, and the rotation angle $\phi$ is determined by the signal constellation $\mathcal{A}$, which is specified in the previous two sections. Note that, the code in (29) is the same as the TBH scheme (15), 


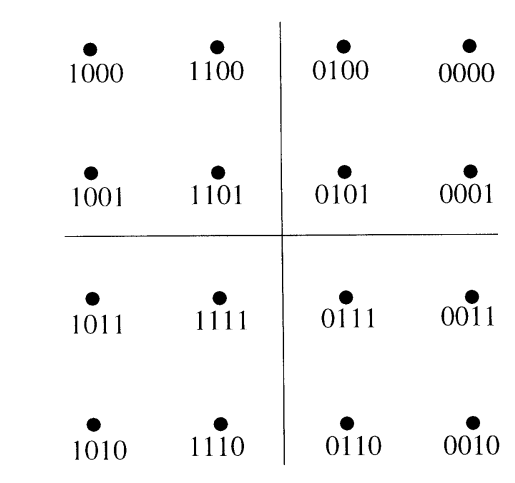

(a) $16-\mathrm{QAM}: d_{\min , \zeta}(\pi / 4)=d_{\min }=0.6325$

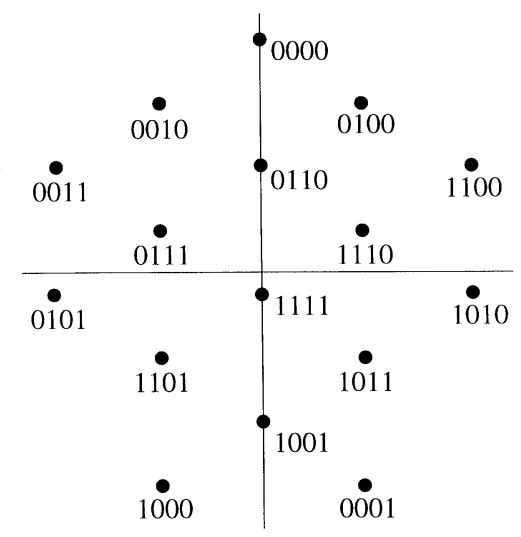

(b) $16-\mathrm{TRI}: d_{\min , \zeta}(\pi / 6)=d_{\min }=0.6761$

Fig. 3. 16-point signal constellations: (a) 16-QAM (b) 16-TRI.

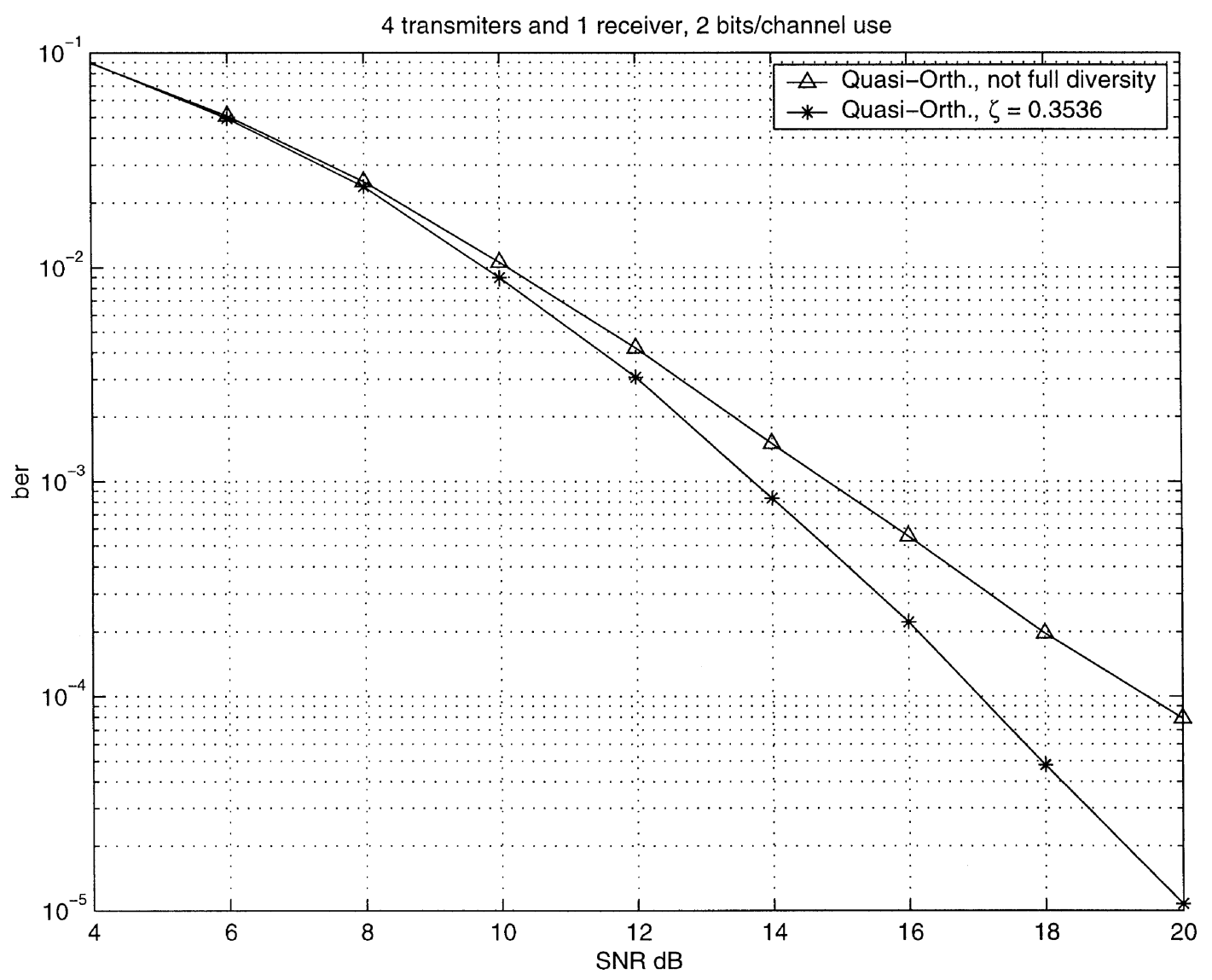

Fig. 4. Performances of the new scheme (line with $*$ ) and the TBH scheme (line with $\triangle$ ).

but the signal constellations here are different from those in the TBH scheme.

First, we compared the new scheme with the TBH scheme (15) in Fig. 4 with a transmission rate of 2 bits per channel use. The signal constellation in both schemes was 4-QAM. The rotation angle $\phi$ was chosen as $\pi / 4$ in the new scheme. Clearly, the performance of the new scheme shows a better diversity order than that of the TBH scheme (15), and the new scheme outperforms the TBH scheme significantly at high SNR. Note that the decoding complexity and the transmission rate of both schemes are the same. Thus, compared with the TBH scheme (15), the new scheme with full diversity gets better performance without extra cost.

We also compared the new scheme with the orthogonal design (10) in Fig. 5 with a transmission rate of 3 bits per channel use. In the new scheme, the signal constellation was 8-QAM, and the rotation angle was $\pi / 4$. We simulated the orthogonal design (10) with both 16-QAM and 16-TRI. We observe that the performance of the TBH scheme is better than that of the orthogonal design at low SNR, but worse at high SNR. We can also see that the new scheme with full diversity further outperforms the orthogonal design at both low and high SNRs. This 


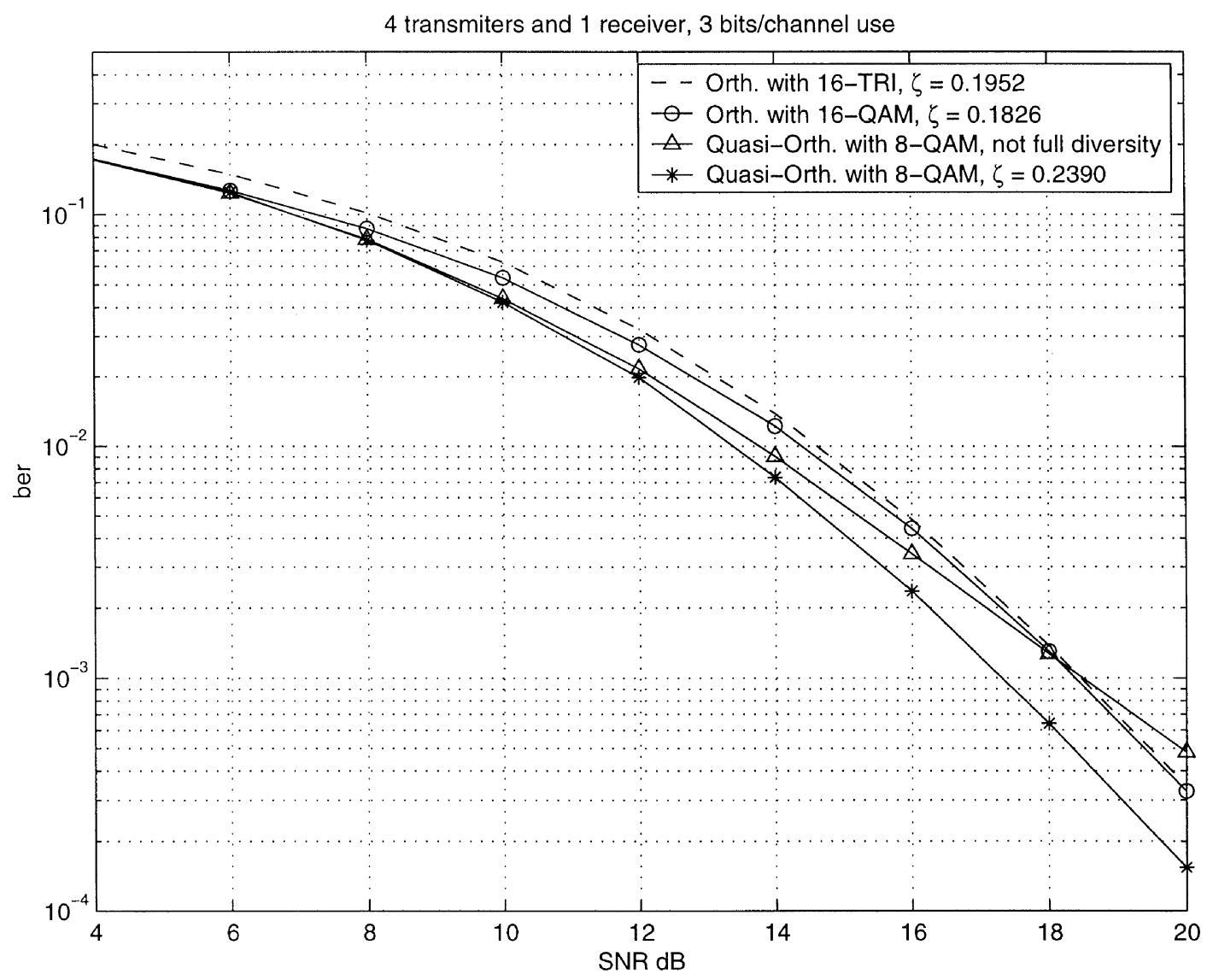

Fig. 5. Performances of the new scheme (line with $*$ ), the TBH scheme (line with $\triangle$ ), and the orthogonal design (line with $\circ$ for 16-QAM and dash line for 16-TRI).

is due to the fact that, the diversity product of the new scheme with 8-QAM is larger than that of the orthogonal design with 16-QAM or 16-TRI.

With different signal constellations, we compared the diversity product between the new scheme and the orthogonal design (10) in Table I. With the same transmission rate, the diversity product of the new scheme is always larger than that of the orthogonal design.

\section{B. For Eight Transmit Antennas}

For eight transmit antennas, the quasi-orthogonal STBC with full diversity is given by

$$
C=\sqrt{\frac{4}{3}}\left[\begin{array}{rrrrrrrr}
x_{1} & x_{2} & x_{3} & 0 & x_{4} & x_{5} & x_{6} & 0 \\
-x_{2}^{*} & x_{1}^{*} & 0 & x_{3} & -x_{5}^{*} & x_{4}^{*} & 0 & x_{6} \\
-x_{3}^{*} & 0 & x_{1}^{*} & -x_{2} & -x_{6}^{*} & 0 & x_{4}^{*} & -x_{5} \\
0 & -x_{3}^{*} & x_{2}^{*} & x_{1} & 0 & -x_{6}^{*} & x_{5}^{*} & x_{4} \\
x_{4} & x_{5} & x_{6} & 0 & x_{1} & x_{2} & x_{3} & 0 \\
-x_{5}^{*} & x_{4}^{*} & 0 & x_{6} & -x_{2}^{*} & x_{1}^{*} & 0 & x_{3} \\
-x_{6}^{*} & 0 & x_{4}^{*} & -x_{5} & -x_{3}^{*} & 0 & x_{1}^{*} & -x_{2} \\
0 & -x_{6}^{*} & x_{5}^{*} & x_{4} & 0 & -x_{3}^{*} & x_{2}^{*} & x_{1}
\end{array}\right]
$$

where $x_{1}, x_{2}, x_{3} \in \mathcal{A}$ and $x_{4}, x_{5}, x_{6} \in e^{j \phi} \mathcal{A}$ for some signal constellation $\mathcal{A}$, and the rotation angle $\phi$ is determined by the signal constellation $\mathcal{A}$, which is specified in the previous sections. The factor $\sqrt{4 / 3}$ in (30) ensures that the quasi-orthogonal STBC obeys the energy constraint, i.e., $E\|C\|_{F}^{2}=64$. If we do not rotate some of the signal constellations, i.e., $\phi=0$, then the performance of the code (30) is the same as that of the Jafarkhani scheme [13]. Here, the new scheme takes the advantage of rotating some of the signal constellations to achieve the full diversity.

We compared the new scheme with the Jafarkhani scheme [13], and also with the orthogonal design (11). Fig. 6 provides simulation results with transmission rate of 1.5 bits per channel use. The signal constellation was 4-QAM, and the rotation angle $\phi$ was $\pi / 4$ in the new scheme. We simulated the orthogonal design (11) with both 8-QAM and 8-TRI. We observe that the performance of the Jafarkhani scheme with 4-QAM is better than that of the orthogonal design with 8-QAM or 8-TRI., and the performance of the new scheme with full diversity is better than that of the Jafarkhani scheme.

With different signal constellations, we calculated the diversity product of the new scheme in Table II, and compared it with that of the orthogonal design (11). We observe that with the same transmission rate, the diversity product of the new scheme is always larger than that of the orthogonal design.

\section{For 16 Transmit Antennas}

For 16 transmit antennas, the quasi-orthogonal STBC with full diversity is given by

$$
C=\sqrt{2}\left[\begin{array}{ll}
A & B \\
B & A
\end{array}\right]
$$




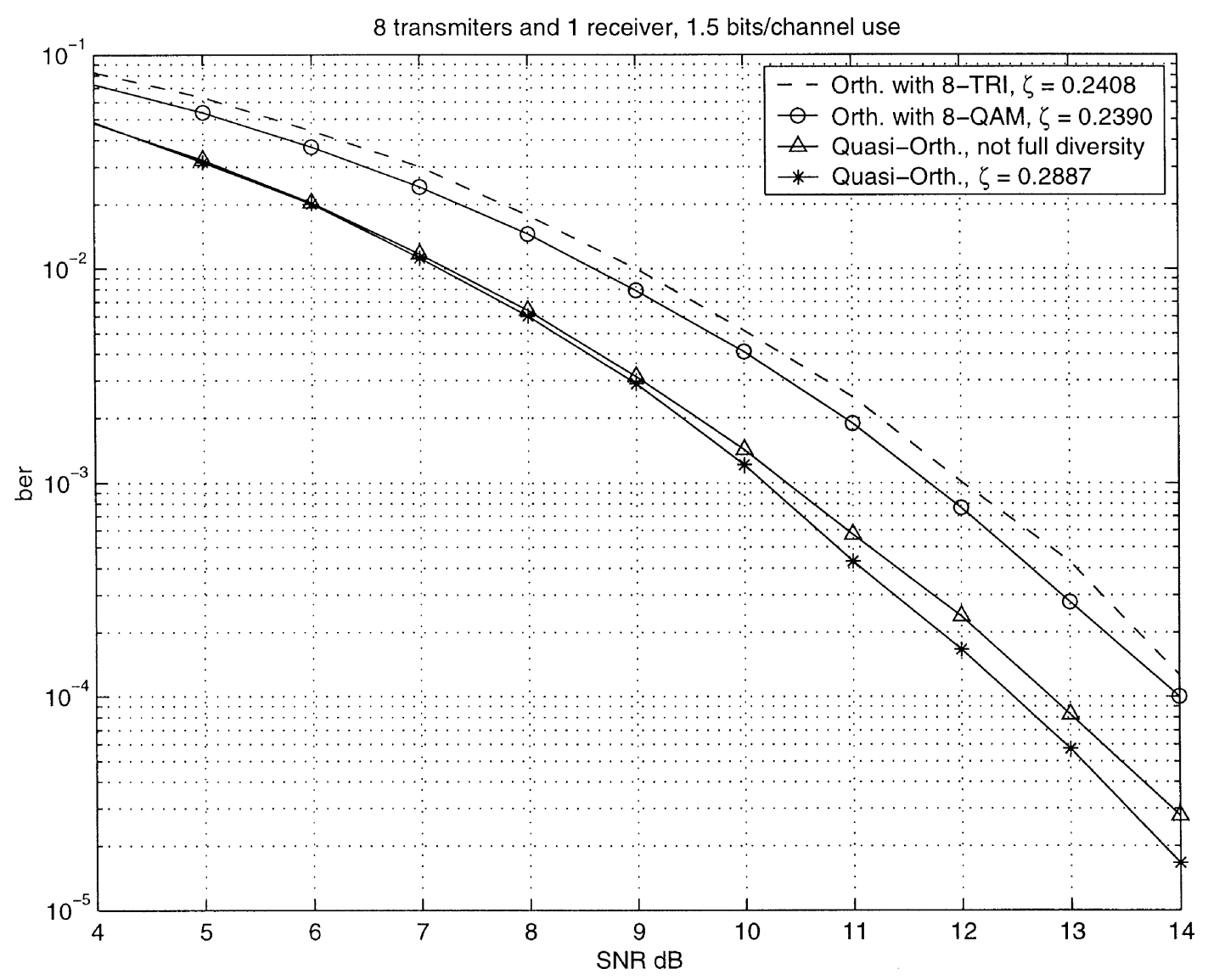

Fig. 6. Performances of the new scheme (line with $*$ ), the Jafarkhani scheme (line with $\triangle$ ), and the orthogonal design (line with o for 8-QAM and dash line for 8-TRI).

where

$$
A=\left[\begin{array}{rrrrrrrr}
x_{1} & x_{2} & x_{3} & 0 & x_{4} & 0 & 0 & 0 \\
-x_{2}^{*} & x_{1}^{*} & 0 & x_{3} & 0 & x_{4} & 0 & 0 \\
-x_{3}^{*} & 0 & x_{1}^{*} & -x_{2} & 0 & 0 & x_{4} & 0 \\
0 & -x_{3}^{*} & x_{2}^{*} & x_{1} & 0 & 0 & 0 & x_{4} \\
-x_{4}^{*} & 0 & 0 & 0 & x_{1}^{*} & -x_{2} & -x_{3} & 0 \\
0 & -x_{4}^{*} & 0 & 0 & x_{2}^{*} & x_{1} & 0 & -x_{3} \\
0 & 0 & -x_{4}^{*} & 0 & x_{3}^{*} & 0 & x_{1} & x_{2} \\
0 & 0 & 0 & -x_{4}^{*} & 0 & x_{3}^{*} & -x_{2}^{*} & x_{1}^{*}
\end{array}\right]
$$

and

$$
B=\left[\begin{array}{rrrrrrrr}
x_{5} & x_{6} & x_{7} & 0 & x_{8} & 0 & 0 & 0 \\
-x_{6}^{*} & x_{5}^{*} & 0 & x_{7} & 0 & x_{8} & 0 & 0 \\
-x_{7}^{*} & 0 & x_{5}^{*} & -x_{6} & 0 & 0 & x_{8} & 0 \\
0 & -x_{7}^{*} & x_{6}^{*} & x_{5} & 0 & 0 & 0 & x_{8} \\
-x_{8}^{*} & 0 & 0 & 0 & x_{5}^{*} & -x_{6} & -x_{7} & 0 \\
0 & -x_{8}^{*} & 0 & 0 & x_{6}^{*} & x_{5} & 0 & -x_{7} \\
0 & 0 & -x_{8}^{*} & 0 & x_{7}^{*} & 0 & x_{5} & x_{6} \\
0 & 0 & 0 & -x_{8}^{*} & 0 & x_{7}^{*} & -x_{6}^{*} & x_{5}^{*}
\end{array}\right]
$$

where $x_{1}, x_{2}, x_{3}, x_{4} \in \mathcal{A}$ and $x_{5}, x_{6}, x_{7}, x_{8} \in e^{j \phi} \mathcal{A}$ for some signal constellation $\mathcal{A}$, and the rotation angle $\phi$ is determined by the signal constellation $\mathcal{A}$, which is specified in the previous sections. The factor $\sqrt{2}$ in (30) ensures that the quasi-orthogonal STBC obeys the energy constraint, i.e., $E\|C\|_{F}^{2}=256$. The symbol transmission rate of (31) is $1 / 2$. We list the diversity product for the new scheme in Table III for some signal constellations.

\section{A General Modulation Scheme With FULL DIVERSITY}

In this section, we propose a more general modulation scheme with full diversity. Recall that the ML decoding of the quasi-orthogonal STBCs can be done separately on each pair of symbols $s_{i}$ and $s_{k+i}$. The basic idea of the new scheme introduced in the previous sections is to choose the signal constellations $\mathcal{A}_{i}$ for $s_{i}$ and $\mathcal{A}_{k+i}$ for $s_{k+i}$ properly to ensure that the modulated signals achieve the full diversity. Specifically, we set $\mathcal{A}_{k+i}=e^{j \phi_{i}} \mathcal{A}_{i}$, and select signals $s_{i} \in \mathcal{A}_{i}$ and $s_{k+i} \in e^{j \phi_{i}} \mathcal{A}_{i}$ independently, i.e., all pairs $\left(s_{i}, s_{k+i}\right) \in \mathcal{A}_{i} \otimes \mathcal{A}_{k+i}$ are taken. As a result, the minimum $\zeta$-distance is upper-bounded by the minimum Euclidean distance of $\mathcal{A}$. A natural question is: can we select the pair of signals $\left(s_{i}, s_{k+i}\right)$ jointly in a four-dimensional (real) signal constellation (or two-dimensional complex signal constellation) to get larger minimum $\zeta$-distance? The answer is positive.

Let us generalize the definition of the minimum $\zeta$-distance at first. Assume that

$\Omega=$

$$
\left\{\left(s_{i, 1}, s_{i, 2}\right): \text { both } s_{i, 1} \text { and } s_{i, 2} \text { are complex, } i=1,2, \ldots, N\right\}
$$

is an $N$-point signal constellation in four dimensions with energy constraint

$$
\frac{1}{N} \sum_{i=1}^{N}\left(\left|s_{i, 1}\right|^{2}+\left|s_{i, 2}\right|^{2}\right)=2 .
$$


TABLE I

SUMMARY OF DIVERSITY PRODUCT FOR ORTHOGONAL AND QUASI-ORTHOGONAL STBCS FOR FOUR TRANSMIT ANTENNAS Note: “Average Bit Errors" Stands for the Average Bit Errors BetweEn the Nearest Two Signals IN THE SignAL CONSTELLATION WiTH BIT LABELING

\begin{tabular}{|c|c|l|l|l|c|}
\hline \multirow{2}{*}{ Constellations } & & \multicolumn{2}{|c|}{ Orth. designs } & \multicolumn{2}{c|}{ Quasi-orth. designs } \\
\cline { 4 - 6 } & Average bit errors & Rate & Diversity product & Rate & Diversity product \\
\hline BPSK & 1 & 0.75 & 0.5774 & 1 & 0.5000 \\
\hline 3-PSK & & 1.19 & 0.5000 & 1.58 & 0.4330 \\
\hline 4-QAM & 1 & 1.5 & 0.4082 & 2 & 0.3536 \\
\hline 5-TRI & & 1.74 & 0.3501 & 2.32 & 0.3032 \\
\hline 6-PSK & & 1.94 & 0.2887 & 2.58 & 0.2500 \\
6-TRI & & 1.94 & 0.3216 & 2.58 & 0.2785 \\
\hline 7-TRI & 1 & 2.11 & 0.3118 & 2.81 & 0.2700 \\
\hline 8-PSK & 1.20 & 2.25 & 0.2209 & 3 & 0.1674 \\
8-QAM & 1.29 & 2.25 & 0.2760 & 3 & 0.2390 \\
8-TRI & 1 & 3 & 0.2780 & 3 & 0.2408 \\
\hline 16-QAM & 1.33 & 3 & 0.1826 & 4 & 0.1581 \\
16-TRI & 1.15 & 3.75 & 0.1291 & 5 & 0.1690 \\
\hline 32-QAM & 1.49 & 3.75 & 0.1377 & 5 & 0.1118 \\
\hline 32-TRI & 1 & 4.5 & 0.0891 & 6 & 0.0772 \\
\hline 64-QAM & 1.41 & 4.5 & 0.0972 & 6 & 0.0842 \\
\hline 64-TRI & & & & & \\
\hline
\end{tabular}

TABLE II

SuMmary OF Diversity PRODUCT FOR ORTHOGONAL AND QUASI-ORTHOGONAL STBCS FOR EIGHT TRANSMIT ANTENNAS. Note: "Average Bit Errors" Stands For the Average Bit Errors BetweEn the NeARest Two Signals IN THE Signal CONSTELLATION WiTH Bit LABELING

\begin{tabular}{|c|c|l|c|l|c|}
\hline & & \multicolumn{2}{|c|}{ Orth. designs } & \multicolumn{2}{c|}{ Quasi-orth. designs } \\
\cline { 3 - 6 } Constellations & Average bit errors & Rate & Diversity product & Rate & Diversity product \\
\hline BPSK & 1 & 0.5 & 0.5000 & 0.75 & 0.4082 \\
\hline 3-PSK & & 0.79 & 0.4330 & 1.19 & 0.3536 \\
\hline 4-QAM & 1 & 1 & 0.3536 & 1.5 & 0.2887 \\
\hline 5-TRI & & 1.16 & 0.3032 & 1.74 & 0.2475 \\
\hline 6-PSK & & 1.29 & 0.2500 & 1.94 & 0.2041 \\
6-TRI & & 1.29 & 0.2785 & 1.94 & 0.2274 \\
\hline 7-TRI & & 1.40 & 0.2700 & 2.11 & 0.2205 \\
\hline 8-PSK & 1 & 1.5 & 0.1913 & 2.25 & 0.1367 \\
8-QAM & 1.20 & 1.5 & 0.2390 & 2.25 & 0.1952 \\
8-TRI & 1.29 & 1.5 & 0.2408 & 2.25 & 0.1966 \\
\hline 16-QAM & 1 & 2 & 0.1581 & 3 & 0.1291 \\
16-TRI & 1.33 & 2 & 0.1690 & 3 & 0.1380 \\
\hline 32-QAM & 1.15 & 2.5 & 0.1118 & 3.75 & 0.0913 \\
32-TRI & 1.49 & 2.5 & 0.1192 & 3.75 & 0.0973 \\
\hline
\end{tabular}

The minimum $\zeta$-distance of the four-dimensional signal constellation $\Omega$ is defined as

$$
d_{\min , \zeta}(\Omega)=\min _{i \neq j}\left|\left(s_{i, 1}-s_{j, 1}\right)^{2}-\left(s_{i, 2}-s_{j, 2}\right)^{2}\right|^{1 / 2}
$$

where $\left(s_{i, 1}, s_{i, 2}\right) \in \Omega$ and $\left(s_{j, 1}, s_{j, 2}\right) \in \Omega$. If we choose the pair of signals $s_{i}$ and $s_{k+i}$ as $\left(s_{i}, s_{k+i}\right) \in \Omega$, then, from (22) we know that the diversity product is determined by the minimum $\zeta$-distance in (32). The proposed scheme in the previous sections is related to the case that the signal constellation $\Omega$ is a tensor product of two two-dimensional signal constellations $\mathcal{A}$ and $\mathcal{B}$, i.e., $\Omega=\mathcal{A} \otimes \mathcal{B}$. It is reasonable to expect that we may get larger minimum $\zeta$-distance if we choose the pair of signals $s_{i}$ and $s_{k+i}$ from a four-dimensional signal constellation $\Omega$. Note that the decoding complexity of the general scheme is the same as that of the proposed scheme in the previous sections. 
TABLE III

SUMMARY OF DIVERSITY PRODUCT FOR THE QUASI-ORTHOGONAL STBCS FOR 16 Transmit ANTENNAS. Note: "AVERAGE Bit ERRORS" STANDS FOR THE Average Bit ERrors Between the NeARest Two Signals in the SIGNAL CONSTELLATION WITH BIT LABELING

\begin{tabular}{|c|c|l|c|}
\hline Constellations & Average bit errors & Rate & Diversity product \\
\hline BPSK & 1 & 0.5 & 0.3536 \\
\hline 4-QAM & 1 & 1 & 0.2500 \\
\hline 8-PSK & 1 & 1.5 & 0.1184 \\
8-QAM & 1.20 & 1.5 & 0.1690 \\
8-TRI & 1.29 & 1.5 & 0.1703 \\
\hline 16-QAM & 1 & 2 & 0.1180 \\
16-TRI & 1.33 & 2 & 0.1195 \\
\hline
\end{tabular}

We present here an example for four-point signal constellations. For convenience, we apply a unitary transform on the four-dimensional signal constellation $\Omega$ as follows:

$$
\left[\begin{array}{l}
x_{i} \\
y_{i}
\end{array}\right]=\frac{1}{\sqrt{2}}\left[\begin{array}{rr}
1 & 1 \\
1 & -1
\end{array}\right] \cdot\left[\begin{array}{l}
s_{i, 1} \\
s_{i, 2}
\end{array}\right], \quad i=1,2, \ldots, N .
$$

Then, $\left\{\left(x_{i}, y_{i}\right): i=1,2, \ldots, N\right\}$ is also a four-dimensional signal constellation with energy constraint

$$
\frac{1}{N} \sum_{i=1}^{N}\left(\left|x_{i}\right|^{2}+\left|y_{i}\right|^{2}\right)=2
$$

and the minimum $\zeta$-distance of $\Omega$ is simplified as

$$
d_{\min , \zeta}(\Omega)=\min _{i \neq j} \sqrt{2}\left|\left(x_{i}-x_{j}\right)\left(y_{i}-y_{j}\right)\right|^{1 / 2} .
$$

Whenever $\left\{\left(x_{i}, y_{i}\right)\right\}_{1 \leq i \leq N}$ is constructed

$$
\left(s_{i}, s_{k+i}\right) \in\left\{\left(s_{i, 1}, s_{i, 2}\right)\right\}_{1 \leq i \leq N}
$$

can then be determined via (33). Now we construct a four-point signal constellation, i.e., $N=4$, as follows (see Fig. 7):

$$
\begin{array}{ll}
x_{1}=y_{1}=e^{i \theta}, & x_{2}=y_{2}=e^{i(\pi-\theta)} \\
x_{3}=y_{4}=e^{i(\pi+\theta)}, & x_{4}=y_{3}=e^{-i \theta}
\end{array}
$$

where $\theta \in[0, \pi / 2]$ is specified as $\sin \theta=\frac{1}{2}(\sqrt{5}-1)$. The minimum $\zeta$-distance of this constellation is $2(\sqrt{5}-1)^{1 / 2}=$ 2.223571. Recall that the minimum $\zeta$-distance for four-point constellation in previously proposed scheme is 2 , in which the constellation is a tensor product of two BPSK constellations.

From Proposition 3 in [26], we know that for a four-point signal constellation in four dimensions, the minimum $\zeta$-distance (34) is upper-bounded by $\frac{4}{3} \sqrt{3}=2.309401$. However, we prove in Appendix $\mathrm{C}$ that this upper bound cannot be reached. We conjecture that the signal constellation in Fig. 7 is optimum in four dimensions from a minimum $\zeta$-distance point of view. Note that the difference between the minimum $\zeta$-distance $2(\sqrt{5}-$ $1)^{1 / 2}$ and the upper bound $\frac{4}{3} \sqrt{3}$ is only 0.085830 . For signal constellations with more than four points in four dimensions, we do not know any other designs with the minimum $\zeta$-distance greater than the scheme we proposed previously.

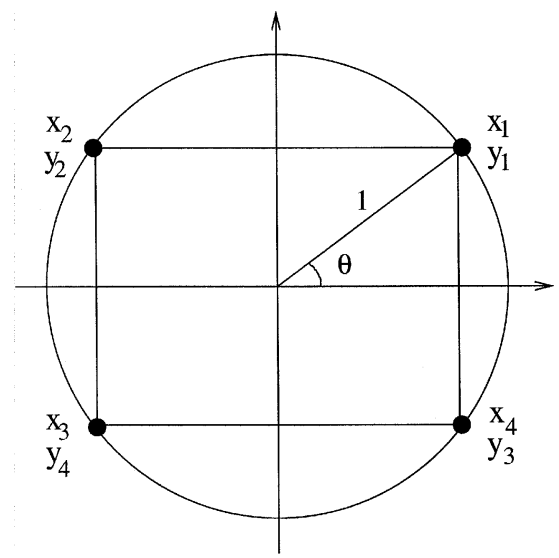

Fig. 7. An example of four-point constellation in four dimensions.

As a remark, using (33), the scheme proposed in the previous sections can be thought of as

$$
\left[\begin{array}{l}
x_{i} \\
y_{i}
\end{array}\right]=\frac{1}{\sqrt{2}}\left[\begin{array}{rr}
1 & 1 \\
1 & -1
\end{array}\right] \cdot\left[\begin{array}{rr}
1 & 0 \\
0 & e^{j \phi}
\end{array}\right]\left[\begin{array}{l}
s_{i, 1} \\
s_{i, 2}
\end{array}\right]
$$

for any $i=1,2, \ldots, N$, where $s_{i, 1}$ and $s_{i, 2}$ belong to a singlesignal constellation $\mathcal{A}$, independently, and $\phi$ can be optimally determined by using the theorems developed in the previous sections. By considering the two $2 \times 2$ matrices in the right-hand side of (35) as two unitary matrices, one might want to ask: is it possible to replace these two unitary matrices by a general unitary matrix $U$ as follows:

$$
\left[\begin{array}{l}
x_{i} \\
y_{i}
\end{array}\right]=U\left[\begin{array}{l}
s_{i, 1} \\
s_{i, 2}
\end{array}\right], \quad i=1,2, \ldots, N
$$

to obtain $\left\{\operatorname{diag}\left(x_{i}, y_{i}\right)\right\}_{1 \leq i \leq N}$ with larger diversity product? The answer is $N O$. It is not hard to show that

$$
\min _{i \neq j} \sqrt{2}\left|\left(x_{i}-x_{j}\right)\left(y_{i}-y_{j}\right)\right|^{1 / 2} \leq d_{\min }(\mathcal{A})
$$

by setting one of $s_{i, l}=s_{j, l}, l=1,2$. Since the upper bound $d_{\min }(\mathcal{A})$ has been reached by the construction as shown in Theorems 1 and 2, the following unitary matrix with the optimum rotation angle $\phi$, as stated in Theorems 1 or 2:

$$
U=\frac{1}{\sqrt{2}}\left[\begin{array}{rr}
1 & 1 \\
1 & -1
\end{array}\right] \cdot\left[\begin{array}{rr}
1 & 0 \\
0 & e^{j \phi}
\end{array}\right]
$$

is already optimum in terms of providing the optimum diversity product from the construction in (36).

Another remark we want to make here is that, after we submitted this paper, we have come across reference [29]. In [29], unitary transformations are used to construct $2^{l} \times 2^{l}$ diagonal STBCs from independent symbols with square lattice (or QAM) signals. We noticed that the transformation in (35) with the rotation angle $\phi=\pi / 4$ found in this paper for the square lattice (or QAM) signal constellations is equivalent to the $2 \times 2$ diagonal code in [27]-[29], which was obtained by a different approach based on the algebraic number fields. However, there is no discussion in [29] dealing with signal constellations from the lattice of equilateral triangles as we presented in Theorem 2, in which the optimum rotation angle $\phi=\pi / 6$ was given. Moreover, the goal of this paper is to design signal constellations for quasi-orthogonal STBCs with full diversity. In the case of 
four $\times 4$ quasi-orthogonal STBC for systems with four transmit antennas and the particular consideration of the tensor-product symbol selection, it turns out that the proposed transformation with the optimum rotation angle is equivalent to the $2 \times 2$ diagonal code in [27]-[29]. However, in general cases, our designs are essentially different from the codes in [27]-[29], as shown in the example in Fig. 7. Some further work in this direction can be found in [41].

\section{CONCLUSION AND FUtURE WORK}

In this paper, we proposed to design the signal constellations properly to ensure that the resulting quasi-orthogonal STBCs can guarantee to achieve the full diversity. We systematically investigated some commonly used signal constellations and determined accordingly the optimum rotation angles in terms of maximizing the diversity product (coding gain). Simulation results showed that the proposed scheme outperforms the STBCs from orthogonal designs at both low and high SNRs. Note that the decoding complexity and the transmission rate of the new scheme are the same as those of the Jafarkhani scheme or the TBH scheme. Therefore, compared with the previous two schemes, the new scheme with full diversity obtains better performance without extra cost. We also proposed a more general modulation scheme with full diversity. However, the results for the general scheme are far from complete. We had only one example of size 4 to stimulate future research.

We would like to point out that, after we submitted this paper, we found that the idea of signal constellation rotations in quasiorthogonal STBCs was also shown independently in [42]-[44], where, however, no optimality of the rotation angle for a general signal constellation was shown. In [42], the optimality of the rotation angle was discussed only for QPSK constellation. In [43], the authors proposed to maximize the metric $\min \left\{\left|x_{i}-x_{j}\right|\right.$, $\left.\left|y_{i}-y_{j}\right|\right\}$, which is a suboptimal approach, while in our proposed scheme, we considered the maximization of the diversity product, i.e., maximizing the metric $\min \left|\left(x_{i}-x_{j}\right)\left(y_{i}-y_{j}\right)\right|$ in (34), which is an optimal approach from the viewpoint of maximizing the coding gain.

\section{APPENDIX A \\ PROOF OF THEOREM 1}

Assume $\mathcal{A}$ is a signal constellation drawn from a square lattice, where the side length of the squares in the lattice is equal to the minimum Euclidean distance of $\mathcal{A}$, denoted as $d_{\min }$. Then, any signal in the constellation $\mathcal{A}$ can be represented as $s_{0}+$ $p_{1} d_{\min }+q_{1} e^{j \pi / 2} d_{\min }$ (see Fig. 8), where $p_{1}$ and $q_{1}$ are integers. Thus, any signal in the rotated constellation $e^{j \pi / 4} \mathcal{A}$ can be represented as $e^{j \pi / 4}\left(s_{0}+p_{2} d_{\text {min }}+q_{2} e^{j \pi / 2} d_{\text {min }}\right)$, where $p_{2}$ and $q_{2}$ are integers.

For any pair of signals $s_{1}$ and $\tilde{s}_{1}$ in $\mathcal{A}$, the difference $s_{1}-\tilde{s}_{1}$ can be represented as

$$
\begin{aligned}
s_{1}-\tilde{s}_{1}= & \left(s_{0}+p_{1} d_{\min }+q_{1} e^{j \pi / 2} d_{\min }\right) \\
& -\left(s_{0}+\tilde{p}_{1} d_{\min }+\tilde{q}_{1} e^{j \pi / 2} d_{\min }\right) \\
= & \left(p_{1}-\tilde{p}_{1}\right) d_{\min }+\left(q_{1}-\tilde{q}_{1}\right) e^{j \pi / 2} d_{\min } .
\end{aligned}
$$

Fig. 8. Representation of the square lattice.

Therefore, for simplicity, we can assume

$$
s_{1}-\tilde{s}_{1}=p_{1} d_{\min }+q_{1} e^{j \pi / 2} d_{\min }
$$

for some integers $p_{1}$ and $q_{1}$. For the same reason, for any pair of signals $s_{2}$ and $\tilde{s}_{2}$ in $e^{j \pi / 4} \mathcal{A}$, we can assume the difference

$$
s_{2}-\tilde{s}_{2}=e^{j \pi / 4}\left(p_{2} d_{\min }+q_{2} e^{j \pi / 2} d_{\min }\right)
$$

for some integers $p_{2}$ and $q_{2}$. Thus, we have

$$
\begin{aligned}
\left|\left(s_{1}-\tilde{s}_{1}\right)^{2}-\left(s_{2}-\tilde{s}_{2}\right)^{2}\right|^{1 / 2} \\
=\mid\left(p_{1} d_{\min }+q_{1} e^{j \pi / 2} d_{\min }\right)^{2} \\
\quad-\left.e^{j \pi / 2}\left(p_{2} d_{\min }+q_{2} e^{j \pi / 2} d_{\min }\right)^{2}\right|^{1 / 2} \\
=\left|\left(p_{1}^{2}-q_{1}^{2}+2 p_{2} q_{2}\right)+\left(q_{2}^{2}-p_{2}^{2}+2 p_{1} q_{1}\right) e^{j \pi / 2}\right|^{1 / 2} d_{\min } \\
=\left[\left(p_{1}^{2}-q_{1}^{2}+2 p_{2} q_{2}\right)^{2}+\left(q_{2}^{2}-p_{2}^{2}+2 p_{1} q_{1}\right)^{2}\right]^{1 / 4} d_{\min } .
\end{aligned}
$$

Since all of $p_{1}, q_{1}, p_{2}$, and $q_{2}$ are integers, so if one of $p_{1}^{2}-q_{1}^{2}+$ $2 p_{2} q_{2}$ and $q_{2}^{2}-p_{2}^{2}+2 p_{1} q_{1}$ is not zero, then

$$
\left(p_{1}^{2}-q_{1}^{2}+2 p_{2} q_{2}\right)^{2}+\left(q_{2}^{2}-p_{2}^{2}+2 p_{1} q_{1}\right)^{2} \geq 1
$$

It follows that

$$
\begin{aligned}
& d_{\min , \zeta}\left(\mathcal{A}, e^{j \pi / 4} \mathcal{A}\right) \\
& =\min _{\left(s_{1}, s_{2}\right) \neq\left(\tilde{s}_{1}, \tilde{s}_{2}\right)}\left|\left(s_{1}-\tilde{s}_{1}\right)^{2}-\left(s_{2}-\tilde{s}_{2}\right)^{2}\right|^{1 / 2} \\
& =\min _{\left(p_{1}, q_{1}, p_{2}, q_{2}\right) \neq 0}\left[\left(p_{1}^{2}-q_{1}^{2}+2 p_{2} q_{2}\right)^{2}\right. \\
& \geq d_{\text {min }} \\
& \left.\quad+\left(q_{2}^{2}-p_{2}^{2}+2 p_{1} q_{1}\right)^{2}\right]^{1 / 4} d_{\text {min }}
\end{aligned}
$$

which is the desired result, since $d_{\min }$ is a upper bound of $d_{\min , \zeta}\left(\mathcal{A}, e^{j \pi / 4} \mathcal{A}\right)$. In the following, we will prove that one of $p_{1}^{2}-q_{1}^{2}+2 p_{2} q_{2}$ and $q_{2}^{2}-p_{2}^{2}+2 p_{1} q_{1}$ is nonzero under the constraint that $\left(s_{1}, s_{2}\right) \neq\left(\tilde{s}_{1}, \tilde{s}_{2}\right)$, i.e., $p_{1}, q_{1}, p_{2}$, and $q_{2}$ cannot be zero at the same time.

If both $p_{1}^{2}-q_{1}^{2}+2 p_{2} q_{2}$ and $q_{2}^{2}-p_{2}^{2}+2 p_{1} q_{1}$ are zeros, i.e.,

$$
\begin{aligned}
& q_{1}^{2}-p_{1}^{2}=2 p_{2} q_{2} \\
& p_{2}^{2}-q_{2}^{2}=2 p_{1} q_{1}
\end{aligned}
$$


then from (37), either both $p_{1}$ and $q_{1}$ are odd, or both $p_{1}$ and $q_{1}$ are even. If both $p_{1}$ and $q_{1}$ are odd, denoted as $p_{1}=2 \tilde{p}_{1}+1$ and $q_{1}=2 \tilde{q}_{1}+1$, then substituting them into (37), we have

$$
4\left(\tilde{q}_{1}^{2}+\tilde{q}_{1}-\tilde{p}_{1}^{2}-\tilde{p}_{1}\right)=2 p_{2} q_{2}
$$

It follows that one of $p_{2}$ and $q_{2}$ is even. On the other hand, from (38) we know that either both $p_{2}$ and $q_{2}$ are odd, or both $p_{2}$ and $q_{2}$ are even. Thus, we conclude that both $p_{2}$ and $q_{2}$ are even, denoted as $p_{2}=2 \tilde{p}_{2}$ and $q_{2}=2 \tilde{q}_{2}$. Substituting them into (38), we have $4\left(\tilde{p}_{2}^{2}-\tilde{q}_{2}^{2}\right)=2 p_{1} q_{1}$, which contradicts the assumption that both $p_{1}$ and $q_{1}$ are odd. So, both $p_{1}$ and $q_{1}$ must be even, denoted as $p_{1}=2 \tilde{p}_{1}$ and $q_{1}=2 \tilde{q}_{1}$

For the same reason, we can prove that both $p_{2}$ and $q_{2}$ must be even, denoted as $p_{2}=2 \tilde{p}_{2}$ and $q_{2}=2 \tilde{q}_{2}$. Substituting $p_{1}=$ $2 \tilde{p}_{1}, q_{1}=2 \tilde{q}_{1}, p_{2}=2 \tilde{p}_{2}$ and $q_{2}=2 \tilde{q}_{2}$ into (37) and (38), we obtain

$$
\begin{aligned}
& \tilde{q}_{1}^{2}-\tilde{p}_{1}^{2}=2 \tilde{p}_{2} \tilde{q}_{2} \\
& \tilde{p}_{2}^{2}-\tilde{q}_{2}^{2}=2 \tilde{p}_{1} \tilde{q}_{1} .
\end{aligned}
$$

Repeating the preceding discussion, we can prove that all of $\tilde{p}_{1}, \tilde{q}_{1}, \tilde{p}_{2}$ and $\tilde{q}_{2}$ are even. Let $\tilde{p}_{1}=2 \tilde{\tilde{p}}_{1}, \tilde{q}_{1}=2 \tilde{\tilde{q}}_{1}, \tilde{p}_{2}=2 \tilde{\tilde{p}}_{2}$, and $\tilde{q}_{2}=2 \tilde{\tilde{q}}_{2}$. Substituting them into (39) and (40), we have

$$
\begin{aligned}
& \tilde{\tilde{q}}_{1}^{2}-\tilde{\tilde{p}}_{1}^{2}=2 \tilde{\tilde{p}}_{2} \tilde{\tilde{q}}_{2} \\
& \tilde{\tilde{p}}_{2}^{2}-\tilde{\tilde{q}}_{2}^{2}=2 \tilde{\tilde{p}}_{1} \tilde{\tilde{q}}_{1} .
\end{aligned}
$$

We can continue this process again and again. Since all of $p_{1}, q_{1}, p_{2}$, and $q_{2}$ are finite integers, so we conclude that some of $p_{1}, q_{1}, p_{2}$ and $q_{2}$ must be zero.

If $p_{1}=0$, then from (38), we have $p_{2}^{2}=q_{2}^{2}$, i.e., $p_{2}= \pm q_{2}$. Substituting $p_{1}=0$ and $p_{2}= \pm q_{2}$ into (37), we have $q_{1}=$ $2 p_{2} q_{2}= \pm 2 q_{2}^{2}$. It follows that $q_{1}=0$ and $q_{2}=0$, since both $q_{1}$ and $q_{2}$ are integers. So all of $p_{1}, q_{1}, p_{2}$, and $q_{2}$ are zeros, which contradicts $\left(s_{1}, s_{2}\right) \neq\left(\tilde{s}_{1}, \tilde{s}_{2}\right)$.

If $q_{1}=0$ or $p_{2}=0$ or $q_{2}=0$, similarly, we can prove that all of $p_{1}, q_{1}, p_{2}$, and $q_{2}$ are zeros, which contradicts $\left(s_{1}, s_{2}\right) \neq$ $\left(\tilde{s}_{1}, \tilde{s}_{2}\right)$.

Thus, we can conclude that one of $p_{1}^{2}-q_{1}^{2}+2 p_{2} q_{2}$ and $q_{2}^{2}-p_{1}^{2}+2 p_{1} q_{1}$ is nonzero under the constraint that $\left(s_{1}, s_{2}\right) \neq$ $\left(\tilde{s}_{1}, \tilde{s}_{2}\right)$. Thus we prove Theorem 1 .

\section{APPENDIX B \\ PROOF OF THEOREM 2}

Assume $\mathcal{A}$ is a signal constellation drawn from a lattice of equilateral triangles, where the side length of the equilateral triangles in the lattice is equal to the minimum Euclidean distance of $\mathcal{A}$, denoted as $d_{\text {min }}$. Then, any signal in the constellation $\mathcal{A}$ can be represented as $s_{0}+p_{1} d_{\text {min }}+q_{1} e^{j \pi / 3} d_{\min }$ (see Fig. 9), where $p_{1}$ and $q_{1}$ are integers. Thus, any signal in the rotated constellation $e^{j \pi / 6} \mathcal{A}$ can be represented as

$$
e^{j \pi / 6}\left(s_{0}+p_{2} d_{\min }+q_{2} e^{j \pi / 3} d_{\min }\right)
$$

where $p_{2}$ and $q_{2}$ are integers.

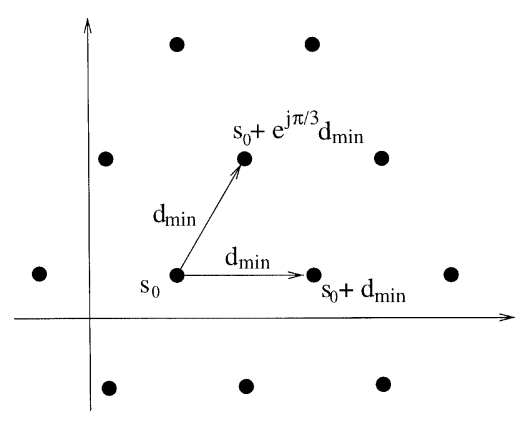

Fig. 9. Representation of the lattice of equilateral triangles.

For any pair of signals $s_{1}$ and $\tilde{s}_{1}$ in $\mathcal{A}$, the difference $s_{1}-\tilde{s}_{1}$ can be represented as

$$
\begin{aligned}
s_{1}-\tilde{s}_{1}= & \left(s_{0}+p_{1} d_{\min }+q_{1} e^{j \pi / 3} d_{\min }\right) \\
& -\left(s_{0}+\tilde{p}_{1} d_{\min }+\tilde{q}_{1} e^{j \pi / 3} d_{\min }\right) \\
= & \left(p_{1}-\tilde{p}_{1}\right) d_{\min }+\left(q_{1}-\tilde{q}_{1}\right) e^{j \pi / 3} d_{\min } .
\end{aligned}
$$

Therefore, for simplicity, we can assume

$$
s_{1}-\tilde{s}_{1}=p_{1} d_{\min }+q_{1} e^{j \pi / 3} d_{\min }
$$

for some integers $p_{1}$ and $q_{1}$. For the same reason, for any pair of signals $s_{2}$ and $\tilde{s}_{2}$ in $e^{j \pi / 6} \mathcal{A}$, we can assume the difference $s_{2}-\tilde{s}_{2}=e^{j \pi / 6}\left(p_{2} d_{\min }+q_{2} e^{j \pi / 3} d_{\min }\right)$ for some integers $p_{2}$ and $q_{2}$. Thus, we have

$$
\begin{aligned}
& \left|\left(s_{1}-\tilde{s}_{1}\right)^{2}-\left(s_{2}-\tilde{s}_{2}\right)^{2}\right|^{1 / 2} \\
& =\mid\left(p_{1} d_{\min }+q_{1} e^{j \pi / 3} d_{\min }\right)^{2} \\
& \quad-\left.e^{j \pi / 3}\left(p_{2} d_{\min }+q_{2} e^{j \pi / 3} d_{\min }\right)^{2}\right|^{1 / 2} \\
& =\mid\left(p_{1}^{2}+q_{2}^{2}\right)+\left(2 p_{1} q_{1}-p_{2}^{2}\right) e^{j \pi / 3} \\
& \quad+\left.\left(q_{1}^{2}-2 p_{2} q_{2}\right) e^{j 2 \pi / 3}\right|^{1 / 2} d_{\min .}
\end{aligned}
$$

For convenience, denote $u=p_{1}^{2}+q_{2}^{2}, v=2 p_{1} q_{1}-p_{2}^{2}$ and $w=q_{1}^{2}-2 p_{2} q_{2}$, then

$$
\begin{aligned}
& \left|\left(s_{1}-\tilde{s}_{1}\right)^{2}-\left(s_{2}-\tilde{s}_{2}\right)^{2}\right|^{1 / 2} \\
& \quad=\left|u+v e^{j \pi / 3}+w e^{j 2 \pi / 3}\right|^{1 / 2} d_{\min } \\
& \quad=\left[\left(u+\frac{1}{2} v-\frac{1}{2} w\right)^{2}+\frac{3}{4}(v+w)^{2}\right]^{1 / 4} d_{\min } \\
& \quad=\left\{\frac{1}{2}\left[(u+v)^{2}+(u-w)^{2}+(v+w)^{2}\right]\right\}^{1 / 4} d_{\min .}
\end{aligned}
$$

Since all of $u, v$ and $w$ are integers, so if two of $u+v, u-w$ and $v+w$ are nonzero, then $(u+v)^{2}+(u-w)^{2}+(v+w)^{2} \geq 2$. It follows that

$$
\begin{aligned}
& d_{\min , \zeta}\left(\mathcal{A}, e^{j \pi / 6} \mathcal{A}\right) \\
& \quad=\min _{\left(s_{1}, s_{2}\right) \neq\left(\tilde{s}_{1}, \tilde{s}_{2}\right)}\left|\left(s_{1}-\tilde{s}_{1}\right)^{2}-\left(s_{2}-\tilde{s}_{2}\right)^{2}\right|^{1 / 2} \\
& \quad=\min _{\left(p_{1}, q_{1}, p_{2}, q_{2}\right) \neq 0}\left\{\frac{1}{2}\left[(u+v)^{2}+(u-w)^{2}+(v+w)^{2}\right]\right\}^{1 / 4} d_{\min } \\
& \quad \geq d_{\min }
\end{aligned}
$$


which is the desired result, since $d_{\min }$ is an upper bound of $d_{\min , \zeta}\left(\mathcal{A}, e^{j \pi / 6} \mathcal{A}\right)$. In the following, we will prove that two of $u+v, u-w$ and $v+w$ are nonzero under the constraint that $\left(s_{1}, s_{2}\right) \neq\left(\tilde{s}_{1}, \tilde{s}_{2}\right)$, i.e., $p_{1}, q_{1}, p_{2}$ and $q_{2}$ cannot be zero at the same time. The proof is divided into three steps.

Step 1: At first, we want to show that if two of $u+v, u-w$, and $v+w$ are zeros, then $u=w=-v$.

If $u+v=0$ and $u-w=0$, then $u=-v$ and $u=w$, i.e., $u=w=-v$; if $u+v=0$ and $v+w=0$, then $u=-v$ and $v=-w$, i.e., $u=w=-v$; if $u-w=0$ and $v+w=0$, then $u=w$ and $v=-w$, i.e., $u=w=-v$.

Step 2: In this step, we show that if $u=w=-v$, then one of $p_{1}, q_{1}, p_{2}$ and $q_{2}$ must be zero.

Suppose that all of $p_{1}, q_{1}, p_{2}$, and $q_{2}$ are not zero. From the assumption $u=w=-v$, we have

$$
p_{1}^{2}+q_{2}^{2}=q_{1}^{2}-2 p_{2} q_{2}=p_{2}^{2}-2 p_{1} q_{1} .
$$

From the second equality in (41), we have

$$
q_{1}^{2}-p_{2}^{2}=2\left(p_{2} q_{2}-p_{1} q_{1}\right) .
$$

Therefore, either both $q_{1}$ and $p_{2}$ are odd, or both $q_{1}$ and $p_{2}$ are even. If both $q_{1}$ and $p_{2}$ are odd, denoted as $q_{1}=2 \tilde{q}_{1}+1$ and $p_{2}=2 \tilde{p}_{2}+1$, then substituting them into (42), we have

$$
4\left(\tilde{q}_{1}^{2}+\tilde{q}_{1}-\tilde{p}_{2}^{2}-\tilde{p}_{2}\right)=2\left[2\left(\tilde{p}_{2} q_{2}-p_{1} \tilde{q}_{1}\right)+\left(q_{2}-p_{1}\right)\right] .
$$

So $q_{2}-p_{1}$ must be even. It follows that

$$
p_{1}^{2}+q_{2}^{2}=\left(p_{1}-q_{2}\right)^{2}+2 p_{1} q_{2}
$$

must be even, which contradicts the fact that, from the first equation in (41), $p_{1}^{2}+q_{2}^{2}=q_{1}^{2}-2 p_{2} q_{2}$ is odd since $q_{1}$ is odd.

From the preceding discussion, we know that both $q_{1}$ and $p_{2}$ must be even, denoted as $q_{1}=2 \tilde{q}_{1}$ and $p_{2}=2 \tilde{p}_{2}$. From the first equation in (41) again, we have

$$
p_{1}^{2}+q_{2}^{2}=4\left(\tilde{q}_{1}^{2}-\tilde{p}_{2} q_{2}\right) .
$$

Therefore, either both $p_{1}$ and $q_{2}$ are odd, or both $p_{1}$ and $q_{2}$ are even. If both $p_{1}$ and $q_{2}$ are odd, denoted as $p_{1}=2 \tilde{p}_{1}+1$ and $q_{2}=2 \tilde{q}_{2}+1$, then substituting them into (43), we have

$$
4\left(\tilde{p}_{1}^{2}+\tilde{p}_{1}+\tilde{q}_{2}^{2}+\tilde{q}_{2}\right)+2=4\left(\tilde{q}_{1}^{2}-\tilde{p}_{2} q_{2}\right)
$$

which is impossible. So both $p_{1}$ and $q_{2}$ must be even, denoted as $p_{1}=2 \tilde{p}_{1}$ and $q_{2}=2 \tilde{q}_{2}$.

By now we know that all of $p_{1}, q_{1}, p_{2}$ and $q_{2}$ are even. Substituting $p_{1}=2 \tilde{p}_{1}, q_{1}=2 \tilde{q}_{1}, p_{2}=2 \tilde{p}_{2}$, and $q_{2}=2 \tilde{q}_{2}$ into (41), we obtain

$$
\tilde{p}_{1}^{2}+\tilde{q}_{2}^{2}=\tilde{q}_{1}^{2}-2 \tilde{p}_{2} \tilde{q}_{2}=\tilde{p}_{2}^{2}-2 \tilde{p}_{1} \tilde{q}_{1}
$$

where all of $\tilde{p}_{1}, \tilde{q}_{1}, \tilde{p}_{2}$, and $\tilde{q}_{2}$ are not zero. Repeating the above discussion, we can prove that all of $\tilde{p}_{1}, \tilde{q}_{1}, \tilde{p}_{2}$ and $\tilde{q}_{2}$ are even. Let $\tilde{p}_{1}=2 \tilde{\tilde{p}}_{1}, \tilde{q}_{1}=2 \tilde{\tilde{q}}_{1}, \tilde{p}_{2}=2 \tilde{\tilde{p}}_{2}$, and $\tilde{q}_{2}=2 \tilde{\tilde{q}}_{2}$. Substituting them into (44), we have

$$
\tilde{\tilde{p}}_{1}^{2}+\tilde{\tilde{q}}_{2}^{2}=\tilde{\tilde{q}}_{1}^{2}-2 \tilde{\tilde{p}}_{2} \tilde{\tilde{q}}_{2}=\tilde{\tilde{p}}_{2}^{2}-2 \tilde{\tilde{p}}_{1} \tilde{\tilde{q}}_{1}
$$

where all of $\tilde{\tilde{p}}_{1}, \tilde{\tilde{q}}_{1}, \tilde{\tilde{p}}_{2}$, and $\tilde{\tilde{q}}_{2}$ are not zero. So we can repeat this process forever. This contradicts the fact that all of $p_{1}, q_{1}, p_{2}$. and $q_{2}$ are finite integers.

Step 3: In this step, we further prove that if $u=w=-v$, then all of $p_{1}, q_{1}, p_{2}$, and $q_{2}$ are zeros.

If $u=w=-v$, from the result of Step 2, we know that one of $p_{1}, q_{1}, p_{2}$, and $q_{2}$ must be zero. If $p_{1}=0$, then according to (41), we have $q_{2}^{2}=q_{1}^{2}-2 p_{2} q_{2}=p_{2}^{2}$. Therefore, either $p_{2}=q_{2}$ or $p_{2}=-q_{2}$. If $p_{2}=-q_{2}$, then

$$
q_{2}^{2}=q_{1}^{2}-2 p_{2} q_{2}=q_{1}^{2}+2 q_{2}^{2}
$$

which implies that $q_{1}=0$ and $q_{2}=0$. If $p_{2}=q_{2}$, then

$$
q_{2}^{2}=q_{1}^{2}-2 p_{2} q_{2}=q_{1}^{2}-2 q_{2}^{2} .
$$

It follow that $q_{1}^{2}=3 q_{2}^{2}$, which also implies that $q_{1}=0$ and $q_{2}=0$, since both $q_{1}$ and $q_{2}$ are integers. We conclude that if $p_{1}=0$, then $p_{2}=0, q_{1}=0$ and $q_{2}=0$, i.e., all of $p_{1}, q_{1}, p_{2}$, and $q_{2}$ are zeros.

If $q_{1}=0$ or $p_{2}=0$ or $q_{2}=0$, similarly, we can prove that all of $p_{1}, q_{1}, p_{2}$, and $q_{2}$ are zeros.

From the above three steps, we know that at least two of $u+v, u-w$, and $v+w$ are nonzero under the constraint that $\left(s_{1}, s_{2}\right) \neq\left(\tilde{s}_{1}, \tilde{s}_{2}\right)$. Thus, we prove Theorem 2 .

\section{APPENDIX C}

Claim: For any four-point signal constellation in four dimensions, the minimum $\zeta$-distance is strictly less than $\frac{4}{3} \sqrt{3}$.

Proof: Assume $\left\{\left(x_{i}, y_{i}\right): i=1,2,3,4\right\}$ is a four-point signal constellation in four dimensions with energy constraint

$$
\frac{1}{4} \sum_{i=1}^{4}\left(\left|x_{i}\right|^{2}+\left|y_{i}\right|^{2}\right)=2 .
$$

According to (34), we have

$$
d_{\min , \zeta}^{2}=\min _{i \neq j} 2\left|\left(x_{i}-x_{j}\right)\left(y_{i}-y_{j}\right)\right| .
$$

Therefore,

$$
\begin{aligned}
6 d_{\min , \zeta}^{2} \leq & \sum_{1 \leq i<j \leq 4} 2\left|\left(x_{i}-x_{j}\right)\left(y_{i}-y_{j}\right)\right| \\
& \leq \sum_{1 \leq i<j \leq 4}^{4}\left(\left|x_{i}-x_{j}\right|^{2}+\left|y_{i}-y_{j}\right|^{2}\right) \\
& =3 \sum_{i=1}^{4}\left(\left|x_{i}\right|^{2}+\left|y_{i}\right|^{2}\right)- \\
& \sum_{1 \leq i<j \leq 4}\left(x_{i} x_{j}^{*}+x_{j} x_{i}^{*}\right)-\sum_{1 \leq i<j \leq 4}\left(y_{i} y_{j}^{*}+y_{j} y_{i}^{*}\right) \\
= & 4 \sum_{i=1}^{4}\left(\left|x_{i}\right|^{2}+\left|y_{i}\right|^{2}\right)-\left|\sum_{i=1}^{4} x_{i}\right|^{2}-\left|\sum_{i=1}^{4} y_{i}\right|^{2}
\end{aligned}
$$

where the last equality follows by

$$
\left|\sum_{i=1}^{4} a_{i}\right|^{2}=\sum_{i=1}^{4}\left|a_{i}\right|^{2}+\sum_{1 \leq i<j \leq 4}\left(a_{i} a_{j}^{*}+a_{j} a_{i}^{*}\right) .
$$


Thus, we have

$$
d_{\min , \zeta}^{2} \leq \frac{2}{3} \sum_{i=1}^{4}\left(\left|x_{i}\right|^{2}+\left|y_{i}\right|^{2}\right)=\frac{16}{3}
$$

i.e., $d_{\min , \zeta} \leq \frac{4}{3} \sqrt{3}$. If $d_{\min , \zeta}=\frac{4}{3} \sqrt{3}$, then we know that the equality holds in both (46) and (47). That the equality holds in (46) means

$$
d_{\min , \zeta}^{2}=2\left|\left(x_{i}-x_{j}\right)\left(y_{i}-y_{j}\right)\right|, \quad \forall 1 \leq i<j \leq 4 .
$$

That the equality holds in (47) means

$$
\left|x_{i}-x_{j}\right|=\left|y_{i}-y_{j}\right|, \quad \forall 1 \leq i<j \leq 4 .
$$

From (49) and (50), we have

$$
\begin{aligned}
\left|x_{1}-x_{2}\right| & =\left|x_{1}-x_{3}\right|=\left|x_{1}-x_{4}\right| \\
& =\left|x_{2}-x_{3}\right|=\left|x_{2}-x_{4}\right|=\left|x_{3}-x_{4}\right|
\end{aligned}
$$

i.e., there are four points $x_{1}, x_{2}, x_{3}$, and $x_{4}$ in two dimensions such that the Euclidean distance between any two points of them is the same. This is impossible. Thus, we conclude that the minimum $\zeta$-distance is strictly less than $\frac{4}{3} \sqrt{3}$.

\section{ACKNOWLEDGMENT}

The authors are grateful to the anonymous reviewers for many valuable comments concerning the presentation.

\section{REFERENCES}

[1] İ, E. Telatar, "Capacity of multi-antenna Gaussian channels," AT\&T Bell Labs., Tech. Rep., 1995.

[2] G. J. Foschini and M. J. Gans, "On limits of wireless communications in a fading environment when using multiple antennas," Wireless Personal Commun., vol. 6, pp. 311-335, Mar. 1998

[3] J.-C. Guey, M. P. Fitz, M. R. Bell, and W.-Y. Kuo, "Signal design for transmitter diversity wireless communication systems over Rayleigh fading channels," IEEE Trans. Commun., vol. 47, pp. 527-537, Apr. 1999.

[4] V. Tarokh, N. Seshadri, and A. R. Calderbank, "Space-time codes for high data rate wireless communication: Performance criterion and code construction," IEEE Trans. Inform. Theory, vol. 44, pp. 744-765, Mar. 1998.

[5] S. Alamouti, "A simple transmit diversity technique for wireless communications," IEEE J. Select. Areas Commun., vol. 16, pp. 1451-1458, Aug. 1998.

[6] V. Tarokh, H. Jafarkhani, and A. R. Calderbank, "Space-time block codes from orthogonal designs," IEEE Trans. Inform. Theory, vol. 45, pp. 1456-1467, July 1999.

[7] 1 , "Correction to 'Space-time block codes from orthogonal designs'," IEEE Trans. Inform. Theory, vol. 46, p. 314, Jan. 2000.

[8] A. R. Hammons Jr. and H. El Gamal, "On the theory of space-time codes for PSK modulation," IEEE Trans. Inform. Theory, vol. 46, pp. 524-542, Mar. 2000.

[9] B. M. Hochwald, T. L. Marzetta, and C. B. Papadias, "A transmitter diversity scheme for wideband CDMA systems based on space-time spreading," IEEE J. Select. Areas Commun., vol. 19, pp. 48-60, Jan. 2001.

[10] B. M. Hochwald and W. Sweldens, "Differential unitary space-time modulation," IEEE Trans. Commun., vol. 48, pp. 2041-2052, Dec. 2000.

[11] B. L. Hughes, "Differential space-time modulation," IEEE Trans. Inform. Theory, vol. 46, pp. 2567-2578, Nov. 2000.

[12] A. Shokrollahi, B. Hassibi, B. M. Hochwald, and W. Sweldens, "Representation theory for high-rate multiple-antenna code design," IEEE Trans. Inform. Theory, vol. 47, pp. 2335-2367, Sept. 2001.
[13] H. Jafarkhani, “A quasi-orthogonal space-time block code," IEEE Trans. Commun., vol. 49, pp. 1-4, Jan. 2001.

[14] G. Ganesan and P. Stoica, "Space-time block codes: A maximum SNR approach," IEEE Trans. Inform. Theory, vol. 47, pp. 1650-1656, May 2001.

[15] O. Tirkkonen and A. Hottinen, "Square-matrix embeddable space-time block codes for complex signal constellations," IEEE Trans. Inform. Theory, vol. 48, pp. 1122-1126, Feb. 2002.

[16] O. Tirkkonen, A. Boariu, and A. Hottinen, "Minimal nonorthogonality rate 1 space-time block code for 3+ Tx antennas," in Proc. IEEE 6th Int. Symp. Spread-Spectrum Techniques and Applications (ISSSTA 2000), Sept. 2000, pp. 429-432.

[17] C. B. Papadias and G. J. Foschini, "Capacity-approaching space-time codes for systems employing four transmitter antennas," IEEE Trans. Inform. Theory, vol. 49, pp. 726-732, Mar. 2003.

[18] W. Su and X.-G. Xia, "Two generalized complex orthogonal space-time block codes of rates $7 / 11$ and $3 / 5$ for 5 and 6 transmit antennas," IEEE Trans. Inform. Theory, vol. 49, pp. 313-316, Jan. 2003.

[19] - "On space-time block codes from complex orthogonal designs," Wireless Personal Commun., vol. 25, no. 1, pp. 1-26, Apr. 2003.

[20] X.-B. Liang, "A high-rate orthogonal space-time block code," IEEE Commun. Lett., vol. 7, May 2003.

[21] _ , "Orthogonal designs with maximal rates," IEEE Trans. Inform. Theory, vol. 49, pp. 2468-2503, Oct. 2003.

[22] W. Su, X.-G. Xia, and K. J. R. Liu, "A systematic design of high-rate complex orthogonal space-time block codes," IEEE Commun. Lett., vol. 8, pp. 380-382, June 2004.

[23] K. Lu, S. Fu, and X.-G. Xia, "Closed form design of complex orthogonal space-time block codes of rates $(k+1) / 2 k$ for $2 k-1$ and $2 k$ transmit antennas," in Proc. Int. Symp. Information Theory (ISIT 2004), Chicago, IL, June/July 2004, p. 307. Submitted for publication in IEEE Trans. Inform. Theory.

[24] X.-B. Liang and X.-G. Xia, "On the nonexistence of rate-one generalized complex orthogonal designs," IEEE Trans. Inform. Theory, vol. 49, pp. 2984-2989, Nov. 2003.

[25] H. Wang and X.-G. Xia, "Upper bounds of rates of complex orthogonal space-time block codes," IEEE Trans. Inform. Theory, vol. 49, pp. 2788-2796, Oct. 2003.

[26] X.-B. Liang and X.-G. Xia, "Unitary signal constellations for differential space-time modulation with two transmit antennas: Parametric codes, optimal designs and bounds," IEEE Trans. Inform. Theory, vol. 48, pp. 2291-2322, Aug. 2002.

[27] X. Giraud, E. Boutillon, and J.-C. Belfiore, "Algebraic tools to build modulation schemes for fading channels," IEEE Trans. Inform. Theory, vol. 43, pp. 938-952, May 1997.

[28] J. Boutros and E. Viterbo, "Signal space diversity: A power- and bandwidth-efficient diversity technique for the Rayleigh fading channel," IEEE Trans. Inform. Theory, vol. 44, pp. 1453-1467, July 1998.

[29] M. O. Damen, K. Abed-Meraim, and J.-C. Belfiore, "Diagonal algebraic space-time block codes," IEEE Trans. Inform. Theory, vol. 48, pp. 628-636, Mar. 2002.

[30] E. Larsson and P. Stoica, Space-Time Block Coding for Wireless Communications. Cambridge, U.K.: Cambridge Univ. Press, 2003.

[31] B. Vucetic and J. Yuan, Space-Time Coding. Chichester, U.K.: Wiley, 2003.

[32] T. Josefiak, "Realization of Hurwitz-Radon matrices," Queen's Papers in Pure and Applied Math., no. 36, pp. 346-351, 1976.

[33] W. Wolfe, "Amicable orthogonal designs-Existence," Canad. J. Math., vol. 28, pp. 1006-1020, 1976.

[34] A. V. Geramita and J. M. Geramita, "Complex orthogonal designs," $J$. Comb. Theory, ser. A, no. 25, pp. 211-225, 1978.

[35] A. V. Geramita and J. Seberry, Orthogonal Designs, Quadratic Forms and Hadamard Matrices (Lecture Notes in Pure and Applied Mathematics). New York: Marcel Dekker, 1979, vol. 43.

[36] D. B. Shapiro, Compositions of Quadratic Forms. New York: De Gruyter, 2000.

[37] M. K. Simon and J. G. Smith, "Hexagonal multiple phase-and-amplitude-shift-keyed signal sets," IEEE Trans. Commun., vol. COM-21, pp. 1108-1115, Oct. 1973.

[38] G. J. Foschini, R. D. Gitlin, and S. B. Weinstein, "Optimization of twodimensional signal constellations in the presence of Gaussian noise," IEEE Trans. Commun., vol. COM-22, pp. 28-38, 1974.

[39] J. H. Conway and N. J. A. Sloane, "A fast encoding method for lattice codes and quantizers," IEEE Trans. Inform. Theory, vol. IT-29, pp. 820-824, Nov. 1983.

[40] G. D. Forney Jr., R. G. Gallager, G. R. Lang, F. M. Longstaff, and S. U. Qureshi, "Efficient modulation for band-limited channels," IEEE J. Select. Areas Commun., vol. JSAC-2, pp. 632-647, Sept. 1984. 
[41] W. Su, Z. Safar, and K. J. R. Liu, "Orthogonal space-time block codes with sphere packing," IEEE Trans. Commun., submitted for publication.

[42] O. Tirkkonen, "Optimizing space-time block codes by constellation rotations," in Proc. Finnish Wireless Communications Workshop FWCW'01, Oct. 2001, pp. 59-60.
[43] N. Sharma and C. Papadias, "Improved quasi-orthogonal codes through constellation rotation," IEEE Trans. Commun., vol. 51, pp. 332-335, Mar. 2003.

[44] H. Jafarkhani, e-mail communications, May 2002. 